\title{
Research Article \\ Explicit Solution of the Inverse Eigenvalue Problem of Real Symmetric Matrices and Its Application to Electrical Network Synthesis
}

\author{
D. B. Kandic ${ }^{1}$ and B. D. Reljin ${ }^{2}$ \\ ${ }^{1}$ Department of Physics E Electrical Engineering, Mechanical Engineering Faculty, \\ University of Belgrade, Kraljice Marije 16, 11120 Belgrade, Serbia \\ ${ }^{2}$ Electrical Engineering Faculty, University of Belgrade, Bulevar Kralja Aleksandra 73, \\ 11000 Belgrade, Serbia \\ Correspondence should be addressed to D. B. Kandić, dbkandic@afrodita.rcub.bg.ac.yu
}

Received 20 January 2008; Accepted 22 May 2008

Recommended by Mohammad Younis

\begin{abstract}
A novel procedure for explicit construction of the entries of real symmetric matrices with assigned spectrum and the entries of the corresponding orthogonal modal matrices is presented. The inverse eigenvalue problem of symmetric matrices with some specific sign patterns (including hyperdominant one) is explicitly solved too. It has been shown to arise thereof a possibility of straightforward solving the inverse eigenvalue problem of symmetric hyperdominant matrices with assigned nonnegative spectrum. The results obtained are applied thereafter in synthesis of driving-point immittance functions of transformerless, common-ground, two-element-kind RLC networks and in generation of their equivalent realizations.
\end{abstract}

Copyright (c) 2008 D. B. Kandić and B. D. Reljin. This is an open access article distributed under the Creative Commons Attribution License, which permits unrestricted use, distribution, and reproduction in any medium, provided the original work is properly cited.

\section{Introduction}

During the past few decades,many papers [1-16] have studied the inverse eigenvalue problems (IEPs) of various types. The solution existence of the specific IEPs was generally considered in $[1,3-8,10,11,13,14]$ without explicit formulation of the corresponding procedure for solution construction, whereas in $[2,9,12,15,16]$ this has been accomplished. The main result of [16] is the proof that IEP of symmetric hyperdominant (hd) matrices with assigned nonnegative spectrum has at least one solution which has also been constructed. This settled an old IEP opened in [17].Hyperdominant matrices have nonnegative diagonal and nonpositive off-diagonal entries and nonnegative hd margins of rows (hd margin of a row is the sum of entries in that row). The tool used in [16] to construct the $n$ th-order hd matrix with assigned spectrum was the $n$ th-order orthogonal Hessenberg matrix constructed as a special product of $n-1$ plane rotations [15]. Hessenberg matrices naturally arise in study 
of symmetric tridiagonal matrices, skew symmetric, and orthogonal matrices $[13,14,18]$. A matrix is upper (lower) Hessenberg if its entry $(k, m)$ vanishes whenever $k>m+1(m>k+1)$.

In practical work, it is commonly assumed to be better not to form Hessenberg matrices explicitly, but to keep them as products of plane rotations. On the other hand, explicit construction of real symmetric matrices with nonnegative spectrum, which either have hd sign pattern or are truly hd, is proved to be an inevitable task in considering the synthesis of driving-point immittance functions of passive, transformerless, common-ground, two element-kind $R L C$ networks and in generation of their equivalent realizations [17-19]. RLC networks are comprised solely of resistors $(R)$, inductors $(L)$, and capacitors $(C)$. Drivingpoint immittance function of a lumped, time invariant, linear electrical network is either a driving-point impedance $Z(s)$, or a driving-point admittance $Y(s)=Z^{-1}(s)(s=\sigma+j \cdot \omega$ is the complex frequency; $\sigma, \omega$ are real numbers; $j:=\sqrt{-1}$ ). It is well known that a real rational function in $s$ can be driving-point immittance function of $R L C$ network if and only if it is positive real function in s; or similarly, a necessary condition for a stable square matrix $\mathbf{W}(s)$ of real rational functions in $s$ to be driving-point immittance matrix of a passive $R L C$ network is that $\mathbf{W}(s)$ be positive real matrix $[20,21]$. A few tests for ascertaining positive real properties of functions and/or matrices can be found in [20,21]. In [22] it has been pointed out the role of hd matrices in synthesis of both passive and active, transformerless, commonground multiports. Unlike [16], this paper presents explicit construction of entries of real symmetric matrices with arbitrarily assigned spectrum and the entries of the corresponding orthogonal modal matrices. It also presents explicit construction of real symmetric matrices with assigned spectrum and with specific sign patterns (including hd one). Thereof, a solution to the IEP of symmetric, truly hd matrices with assigned nonnegative spectrum is produced. Some of the obtained results are then applied in synthesis of driving-point immitances of transformerless, common-ground, two-element-kind RLC networks and in generation of their equivalent realizations. The two proposed realization procedures are illustrated by an example.Throughout the paper $\oplus$ denotes direct sum, $\mathbf{x}^{T}$ denotes transpose of $\mathbf{x}$, bold capital letters denote matrices and $\mathbf{I}_{k}$ stands for the $k$ th-order unit or identity matrix.

\section{Explicit solution to the IEP of real symmetric matrices by using canonic orthogonal transformations}

Let $\left\{\lambda_{1}, \lambda_{2}, \ldots, \lambda_{n}\right\}$ be assigned spectrum of the sought real symmetric matrices and let $\mathrm{G}_{1}:=$ $\operatorname{diag}\left(\lambda_{1}, \lambda_{2}, \ldots, \lambda_{n}\right)$ be $n \times n$ spectral matrix. Consider a set of $2 \times 2$ orthogonal matrices $\mathbf{P}_{k} \in$ $\left\{\mathbf{A}_{k}, \mathbf{B}_{k}, \mathbf{C}_{k}, \mathbf{D}_{k}\right\}(k=1, \ldots, n-1)$ :

$$
\begin{aligned}
\mathbf{P}_{k} & :=\left[\begin{array}{ll}
a_{k} & b_{k} \\
c_{k} & d_{k}
\end{array}\right], \quad \mathbf{A}_{k}:=\left[\begin{array}{cc}
\cos \theta_{k} & -\sin \theta_{k} \\
\sin \theta_{k} & \cos \theta_{k}
\end{array}\right], \quad \mathbf{B}_{k}:=\left[\begin{array}{cc}
\cos \theta_{k} & \sin \theta_{k} \\
\sin \theta_{k} & -\cos \theta_{k}
\end{array}\right], \\
\mathbf{C}_{k} & :=\left[\begin{array}{cc}
\cos \theta_{k} & \sin \theta_{k} \\
-\sin \theta_{k} & \cos \theta_{k}
\end{array}\right], \quad \mathbf{D}_{k}:=\left[\begin{array}{cc}
-\cos \theta_{k} & \sin \theta_{k} \\
\sin \theta_{k} & \cos \theta_{k}
\end{array}\right], \quad \theta_{k} \in\left[0, \frac{\pi}{2}\right],
\end{aligned}
$$

which are either rotators $\left(\mathbf{A}_{k}\right.$ and $\left.\mathbf{C}_{k}\right)$ or reflectors $\left(\mathbf{B}_{k}\right.$ and $\left.\mathbf{D}_{k}\right)$. A useful set of $n \times n$ orthogonal matrices is

$$
\mathbf{U}_{1}:=\mathbf{P}_{1} \oplus \mathbf{I}_{n-2}, \quad \mathbf{U}_{k}:=\mathbf{I}_{k-1} \oplus \mathbf{P}_{k} \oplus \mathbf{I}_{n-k-1} \quad(k=2, \ldots, n-2), \quad \mathbf{U}_{n-1}:=\mathbf{I}_{n-2} \oplus \mathbf{P}_{n-1} .
$$


From the following two matrix recurrent relations

$$
\mathbf{G}_{k+1}:=\mathbf{U}_{k} \mathbf{G}_{k} \mathbf{U}_{k^{\prime}}^{\mathrm{T}} \quad \mathbf{S}_{k+1}:=\mathbf{U}_{n-k} \mathbf{S}_{k} \mathbf{U}_{n-k}^{\mathrm{T}} \quad(k=1, \ldots, n-1),
$$

we readily obtain $n \times n$ real symmetric matrices $\mathbf{G}_{n}$ and $\mathbf{S}_{n}$, which are both congruent and similar to $\mathrm{G}_{1}$

$$
\mathbf{G}_{n}=\mathbf{U G}_{1} \mathbf{U}^{\mathrm{T}}, \quad \mathbf{U}:=\mathbf{U}_{n-1} \mathbf{U}_{n-2} \cdots \mathbf{U}_{1} ; \quad \mathbf{S}_{n}=\mathbf{V G}_{1} \mathbf{V}^{\mathrm{T}}, \quad \mathbf{V}:=\mathbf{V}_{1} \mathbf{V}_{2} \cdots \mathbf{V}_{n-1}
$$

Columns of the orthogonal modal matrix $\mathbf{U}(\mathbf{V})$ correspond to eigenvectors of $\mathbf{G}_{n}\left(\mathbf{S}_{n}\right)$. Out of $(n-1)$ ! different possibilities of using (2.3) in generation of $\mathbf{G}_{n}$ and $\mathbf{S}_{n}$, only the two selected by (2.4) produce explicit expressions of entries of $\mathbf{G}_{n}$ and $\mathbf{S}_{n}$ in terms of $\left\{\lambda_{1}, \lambda_{2}, \ldots, \lambda_{n}\right\}$ and the entries of $\mathbf{P}_{k}(k=1, \ldots, n-1)$. $\mathbf{U}(\mathbf{V})$ from (2.4) will be shown later to take on lower (upper) Hessenberg form with the entries explicitly expressed too. For the sake of brevity, we will restrict our consideration only to the first of relations (2.3), bearing on mind the possibility of treating the second one similarly. For $k=1$ and $k=2$ we readily obtain $\mathbf{G}_{2}$ and $\mathbf{G}_{3}$, by using (2.1) and (2.3):

$$
\begin{aligned}
& \mathbf{G}_{2}=\left(\mathbf{P}_{1} \oplus \mathbf{I}_{n-2}\right) \mathbf{G}_{1}\left(\mathbf{P}_{1}^{\mathrm{T}} \oplus \mathbf{I}_{n-2}\right) \\
& =\left[\begin{array}{c|c|c}
a_{1} & b_{1} & \mathbf{0}_{2, n-2} \\
\hline c_{1} & d_{1} & \\
\hline \mathbf{0}_{2, n-2}^{\mathrm{T}} & \mathbf{I}_{n-2}
\end{array}\right]\left[\begin{array}{c|c|ccc|c}
\lambda_{1} & 0 & & & \mathbf{0}_{2, n-2} & \\
\hline 0 & \lambda_{2} & & & \\
\hline & \lambda_{3} & & \\
& & & \mathbf{0} & \\
\mathbf{0}_{2, n-2}^{\mathrm{T}} & & \ddots & \\
& & \mathbf{0} & & \\
\hline & & & & \lambda_{n}
\end{array}\right]\left[\begin{array}{c|c|c|c}
a_{1} & c_{1} & \mathbf{0}_{2, n-2} \\
\hline b_{1} & d_{1} & \\
\hline \mathbf{0}_{2, n-2}^{\mathrm{T}} & \mathbf{I}_{n-2}
\end{array}\right]
\end{aligned}
$$

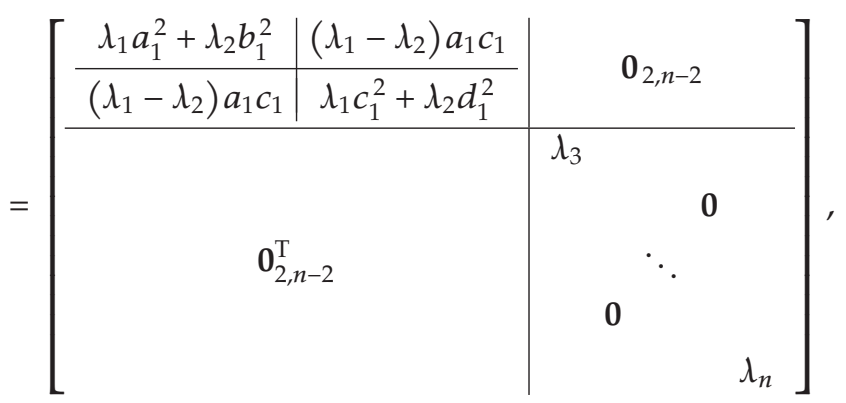

$$
\left\{\begin{array}{c}
\text { More generally, } \forall k=1, \ldots, n-1 \text { it holds } \\
a_{k} b_{k}+c_{k} d_{k}=a_{k} c_{k}+b_{k} d_{k}=0 \\
a_{k}^{2}+b_{k}^{2}=c_{k}^{2}+d_{k}^{2}=a_{k}^{2}+c_{k}^{2}=b_{k}^{2}+d_{k}^{2}=1
\end{array}\right\}
$$




$$
\begin{aligned}
& \mathbf{G}_{3}=\left(1 \oplus \mathbf{P}_{2} \oplus \mathbf{I}_{n-3}\right) \mathbf{G}_{2}\left(1 \oplus \mathbf{P}_{2}^{\mathrm{T}} \oplus \mathbf{I}_{n-3}\right)
\end{aligned}
$$

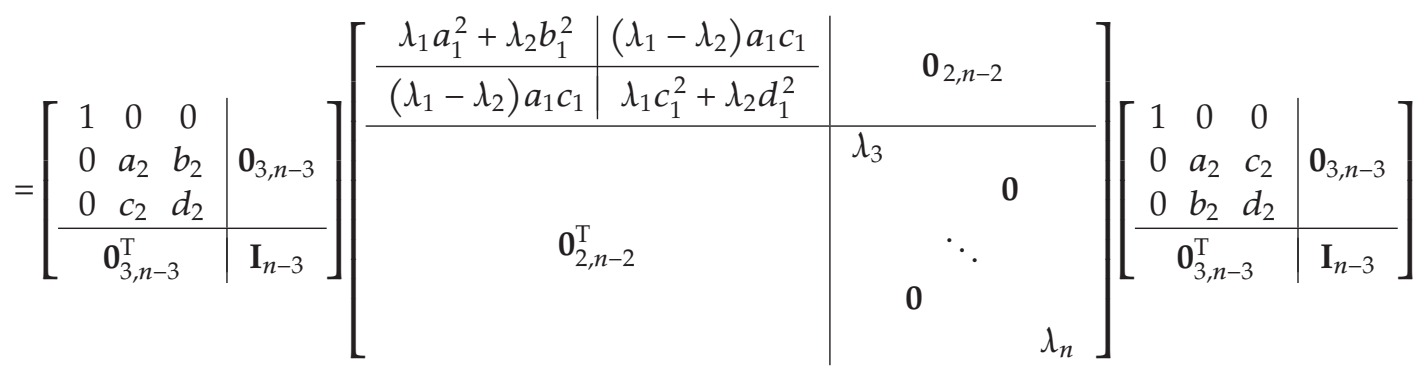

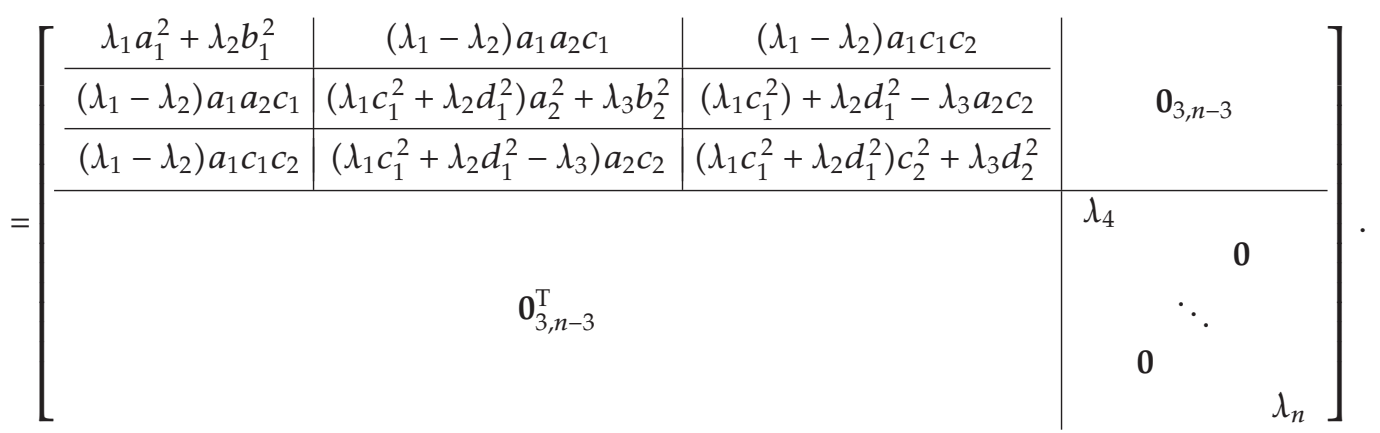

Let $\lambda_{1}^{*}:=\lambda_{1}, \varepsilon_{1}:=\left(\lambda_{1}^{*}-\lambda_{2}\right) a_{1}$, and $\mathbf{x}_{2}:=c_{1} \varepsilon_{1}$, and let us firstly introduce in (2.5) the following notation:

$$
\begin{gathered}
\mathbf{M}_{2}:=\lambda_{1} a_{1}^{2}+\lambda_{2} b_{1}^{2}, \quad \lambda_{2}^{*}:=\lambda_{1}^{*} c_{1}^{2}+\lambda_{2} d_{1}^{2}, \quad \mathbf{D}_{2}^{*}:=\operatorname{diag}\left(\lambda_{2}^{*}, \lambda_{3}\right), \quad \mathbf{D}_{2}:=\operatorname{diag}\left(\lambda_{4}, \ldots, \lambda_{n}\right), \\
\mathbf{A}_{2}:=\left[\begin{array}{lll}
\mathbf{x}_{2} \mid & 0
\end{array}\right]^{\mathrm{T}}, \quad \mathbf{M}_{3}:=\left[\begin{array}{c|c}
\lambda_{1} a_{1}^{2}+\lambda_{2} b_{1}^{2} & \left(\lambda_{1}-\lambda_{2}\right) a_{1} a_{2} c_{1} \\
\hline\left(\lambda_{1}-\lambda_{2}\right) a_{1} a_{2} c_{1} & \left(\lambda_{1} c_{1}^{2}+\lambda_{2} d_{1}^{2}\right) a_{2}^{2}+\lambda_{3} b_{2}^{2}
\end{array}\right], \\
\lambda_{3}^{*}:=\left(\lambda_{1} c_{1}^{2}+\lambda_{2} d_{1}^{2}\right) c_{2}^{2}+\lambda_{3} d_{2}^{2}, \quad \mathbf{D}_{3}^{*}:=\operatorname{diag}\left(\lambda_{3}^{*}, \lambda_{4}\right), \\
\mathbf{D}_{3}:=\operatorname{diag}\left(\lambda_{5}, \ldots, \lambda_{n}\right), \quad \mathbf{A}_{3}:=\left[\begin{array}{c|c}
\left(\lambda_{1}-\lambda_{2}\right) a_{1} c_{1} c_{2} & \left(\lambda_{1} c_{1}^{2}+\lambda_{2} d_{1}^{2}-\lambda_{3}\right) a_{2} c_{2} \\
\hline 0 & 0
\end{array}\right] .
\end{gathered}
$$

Thereafter, observing the partition of $\mathbf{G}_{2}$ and $\mathbf{G}_{3}$ obtained in (2.5)

$$
\mathbf{G}_{2}=\left[\begin{array}{c|c|c}
\mathbf{M}_{2} & \mathbf{A}_{2}^{\mathrm{T}} & \mathbf{0}_{1, n-3} \\
\hline \mathbf{A}_{2} & \mathbf{D}_{2}^{*} & \mathbf{0}_{2, n-3} \\
\hline \mathbf{0}_{1, n-3}^{\mathrm{T}} & \mathbf{0}_{2, n-3}^{\mathrm{T}} & \mathbf{D}_{2}
\end{array}\right], \quad \mathbf{G}_{3}=\left[\begin{array}{c|c|c}
\mathbf{M}_{3} & \mathbf{A}_{3}^{\mathrm{T}} & \mathbf{0}_{2, n-4} \\
\hline \mathbf{A}_{3} & \mathbf{D}_{3}^{*} & \mathbf{0}_{2, n-4} \\
\hline \mathbf{0}_{2, n-4}^{\mathrm{T}} & \mathbf{0}_{2, n-4}^{\mathrm{T}} & \mathbf{D}_{3}
\end{array}\right],
$$

it can readily be anticipated the partition of subsequent matrices $\mathbf{G}_{k}(k=4, \ldots, n-2)$ as follows:

$$
\mathbf{G}_{k}=\left[\begin{array}{c|c|c}
\mathbf{M}_{k} & \mathbf{A}_{k}^{\mathrm{T}} & \mathbf{0}_{k-1, n-k-1} \\
\hline \mathbf{A}_{k} & \mathbf{D}_{k}^{*} & \mathbf{0}_{2, n-k-1} \\
\hline \mathbf{0}_{k-1, n-k-1}^{\mathrm{T}} & \mathbf{0}_{2, n-k-1}^{\mathrm{T}} & \mathbf{D}_{k}
\end{array}\right], \quad \mathbf{x}_{k}:=\left[\begin{array}{ll}
x_{k, 1} & x_{k, 2} \cdots x_{k, k-1}
\end{array}\right], \quad \mathbf{A}_{k}:=\left[\begin{array}{c}
\mathbf{x}_{k} \\
\mathbf{0}_{1, k-1}
\end{array}\right],
$$


where $\mathbf{M}_{k}$ is the symmetric $(k-1) \times(k-1)$ matrix, $\mathbf{x}_{k}$ is $1 \times(k-1)$ row vector, $\mathbf{A}_{k}$ is $2 \times(k-1)$ matrix, $\lambda_{k}^{*}$ is modified eigenvalue $\lambda_{k}, \mathbf{D}_{k}^{*}:=\operatorname{diag}\left(\lambda_{k}^{*}, \lambda_{k+1}\right)$ and $\mathbf{D}_{k}:=\operatorname{diag}\left(\lambda_{k+2}, \ldots, \lambda_{n}\right)$. For $k=2, \ldots, n-3$ from $(2.1)-(2.3),(2.8)$ it follows that

$$
\begin{aligned}
& \mathbf{G}_{k+1}=\left(\mathbf{I}_{k-1} \oplus \mathbf{P}_{k} \oplus \mathbf{I}_{n-k-1}\right) \mathbf{G}_{k}\left(\mathbf{I}_{k-1} \oplus \mathbf{P}_{k}^{\mathrm{T}} \oplus \mathbf{I}_{n-k-1}\right)=\left[\begin{array}{c|c|c}
\mathbf{M}_{k} & \mathbf{A}_{k}^{\mathrm{T}} \mathbf{P}_{k}^{\mathrm{T}} & \mathbf{0}_{k-1, n-k-1} \\
\hline \mathbf{P}_{k} \mathbf{A}_{k} & \mathbf{P}_{k} \mathbf{D}_{k}^{*} \mathbf{P}_{k}^{\mathrm{T}} & \mathbf{0}_{2, n-k-1} \\
\hline \mathbf{0}_{k-1, n-k-1}^{\mathrm{T}} & \mathbf{0}_{2, n-k-1}^{\mathrm{T}} & \mathbf{D}_{k}
\end{array}\right], \\
& \mathbf{P}_{k} \mathbf{A}_{k}=\left[\begin{array}{l|l|l|l|}
a_{k} x_{k, 1} & \cdots & a_{k} x_{k, k-1} \\
\hline c_{k} x_{k, 1} & \cdots & c_{k} x_{k, k-1}
\end{array}\right], \quad \mathbf{P}_{k} \mathbf{D}_{k}^{*} \mathbf{P}_{k}^{\mathrm{T}}=\left[\begin{array}{cc|c}
\lambda_{k}^{*} a_{k}^{2}+\lambda_{k+1} b_{k}^{2} & \left(\lambda_{k}^{*}-\lambda_{k+1}\right) a_{k} c_{k} \\
\hline\left(\lambda_{k}^{*}-\lambda_{k+1}\right) a_{k} c_{k} & \lambda_{k}^{*} c_{k}^{2}+\lambda_{k+1} d_{k}^{2}
\end{array}\right] .
\end{aligned}
$$

For $k=2, \ldots, n-3$, let us define: $\lambda_{k+1}^{*}:=\lambda_{k}^{*} c_{k}^{2}+\lambda_{k+1} d_{k}^{2}, \varepsilon_{k}:=\left(\lambda_{k}^{*}-\lambda_{k+1}\right) a_{k}, \psi_{k k}:=\lambda_{k}^{*} a_{k}^{2}+\lambda_{k+1} b_{k}^{2}$ and thereafter $\mathbf{D}_{k+1}:=\operatorname{diag}\left(\lambda_{k+3}, \ldots, \lambda_{n}\right)$ and $\mathbf{D}_{k+1}^{*}:=\operatorname{diag}\left(\lambda_{k}^{*} c_{k}^{2}+\lambda_{k+1} d_{k}^{2}, \lambda_{k+2}\right)$. Then, from (2.8)-(2.9) it follows the identification

$$
\mathbf{M}_{k+1}:=\left[\begin{array}{c|c}
\mathbf{M}_{k} & a_{k} \mathbf{x}_{k}^{\mathrm{T}} \\
\hline a_{k} \mathbf{x}_{k} & \psi_{k k}
\end{array}\right], \quad \mathbf{A}_{k+1}=\left[\begin{array}{c}
\mathbf{x}_{k+1} \\
\mathbf{0}_{1, k}
\end{array}\right]:=\left[\begin{array}{c|c}
c_{k} \mathbf{x}_{k} & c_{k} \varepsilon_{k} \\
\hline \mathbf{0}_{1, k} & 0
\end{array}\right]=\left[\begin{array}{c|c|c|c}
c_{k} x_{k, 1} & \cdots & c_{k} x_{k, k-1} & c_{k} \varepsilon_{k} \\
\hline 0 & \cdots & 0 & 0
\end{array}\right],
$$

which enables the partition of $\mathbf{G}_{k+1}$ in (2.9) to be like that of $\mathbf{G}_{k}$ in (2.8), and that partition of $\mathbf{x}_{k+1}$ be rather simple

$$
\mathbf{G}_{k+1}=\left[\begin{array}{c|c|c}
\mathbf{M}_{k+1} & \mathbf{A}_{k+1}^{\mathrm{T}} & \mathbf{0}_{k, n-k-2} \\
\hline \mathbf{A}_{k+1} & \mathbf{D}_{k+1}^{*} & \mathbf{0}_{2, n-k-2} \\
\hline \mathbf{0}_{k, n-k-2}^{\mathrm{T}} & \mathbf{0}_{2, n-k-2}^{\mathrm{T}} & \mathbf{D}_{k+1}
\end{array}\right], \quad \mathbf{x}_{k+1}:=c_{k}\left[\mathbf{x}_{k} \mid \varepsilon_{k}\right], \quad k=2, \ldots, n-3 .
$$

Let $\psi_{11}:=\mathbf{M}_{2}$. Having uncovered the partition pattern of $\mathbf{M}_{k+1}(k=2, \ldots, n-3)$, we can pursue partitioning of $\mathbf{M}_{n-2}$ backwardly from $\mathbf{M}_{n-2}$ to $\mathbf{M}_{2}$, by using (2.10). Afterwards, we can produce $\mathbf{G}_{n-2}$, by using (2.10)-(2.11). The results are

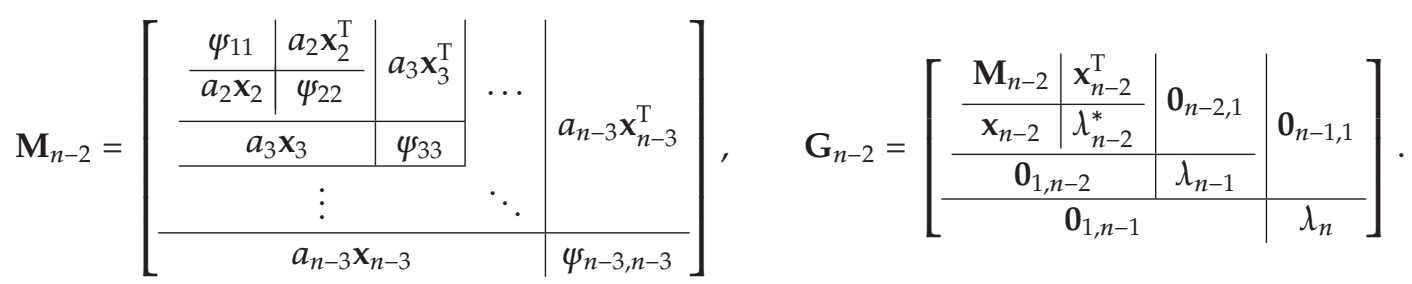

Since $\mathbf{G}_{n-1}:=\left(\mathbf{I}_{n-3} \oplus \mathbf{P}_{n-2} \oplus 1\right) \mathbf{G}_{n-2}\left(\mathbf{I}_{n-3} \oplus \mathbf{P}_{n-2}^{\mathrm{T}} \oplus 1\right)$ and

$$
\mathbf{P}_{n-2}\left[\begin{array}{cc}
\lambda_{n-2}^{*} & 0 \\
0 & \lambda_{n-1}
\end{array}\right] \mathbf{P}_{n-2}^{\mathrm{T}}=\left[\begin{array}{c|c}
\lambda_{n-2}^{*} a_{n-2}^{2}+\lambda_{n-1} b_{n-2}^{2} & \left(\lambda_{n-2}^{*}-\lambda_{n-1}\right) a_{n-2} c_{n-2} \\
\hline\left(\lambda_{n-2}^{*}-\lambda_{n-1}\right) a_{n-2} c_{n-2} & \lambda_{n-2}^{*} c_{n-2}^{2}+\lambda_{n-1} d_{n-2}^{2}
\end{array}\right],
$$


then after defining $\psi_{n-2, n-2}:=\lambda_{n-2}^{*} a_{n-2}^{2}+\lambda_{n-1} b_{n-2}^{2}, \varepsilon_{n-2}:=\left(\lambda_{n-2}^{*}-\lambda_{n-1}\right) a_{n-2}, \lambda_{n-1}^{*}:=\lambda_{n-2}^{*} c_{n-2}^{2}+$ $\lambda_{n-1} d_{n-2}^{2}$ and $\mathbf{x}_{n-1}:=c_{n-2}\left[\mathbf{x}_{n-2} \mid \varepsilon_{n-2}\right]$, it follows from (2.12)-(2.13)

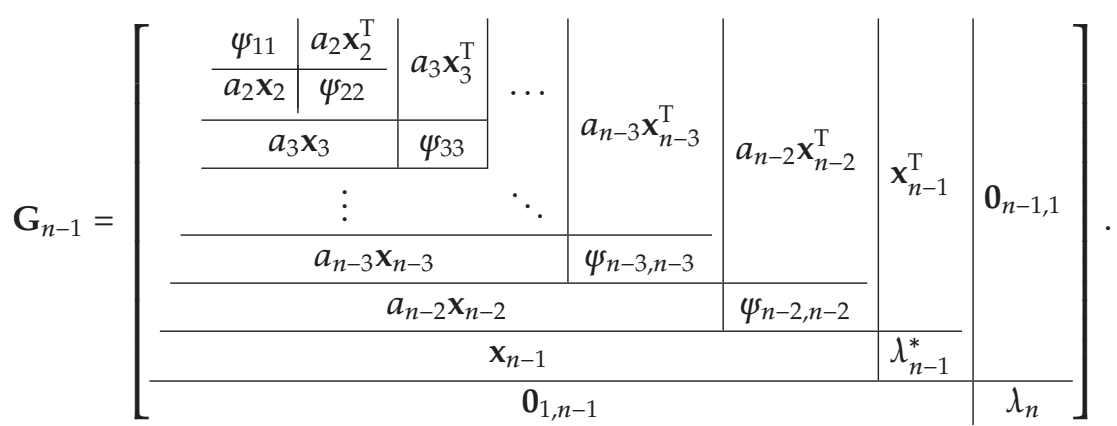

Since $\mathbf{G}_{n}:=\left(\mathbf{I}_{n-2} \oplus \mathbf{P}_{n-1}\right) \mathbf{G}_{n-1}\left(\mathbf{I}_{n-2} \oplus \mathbf{P}_{n-1}^{\mathrm{T}}\right)$ and

$$
\mathbf{P}_{n-1}\left[\begin{array}{cc}
\lambda_{n-1}^{*} & 0 \\
0 & \lambda_{n}
\end{array}\right] \mathbf{P}_{n-1}^{\mathrm{T}}=\left[\begin{array}{c|c}
\lambda_{n-1}^{*} a_{n-1}^{2}+\lambda_{n} b_{n-1}^{2} & \left(\lambda_{n-1}^{*}-\lambda_{n}\right) a_{n-1} c_{n-1} \\
\hline\left(\lambda_{n-1}^{*}-\lambda_{n}\right) a_{n-1} c_{n-1} & \lambda_{n-1}^{*} c_{n-1}^{2}+\lambda_{n} d_{n-1}^{2}
\end{array}\right],
$$

then on introducing $\psi_{n-1, n-1}:=\lambda_{n-1}^{*} a_{n-1}^{2}+\lambda_{n} b_{n-1}^{2}, \varepsilon_{n-1}:=\left(\lambda_{n-1}^{*}-\lambda_{n}\right) a_{n-1}$, and $\lambda_{n}^{*}:=\lambda_{n-1}^{*} c_{n-1}^{2}+$ $\lambda_{n} d_{n-1}^{2}$, we obtain from (2.14)-(2.15) the partition of $\mathbf{G}_{n}$ which is amenable to the production of its entries in explicit form and is suitable for further discussion about solving some specific IEPs

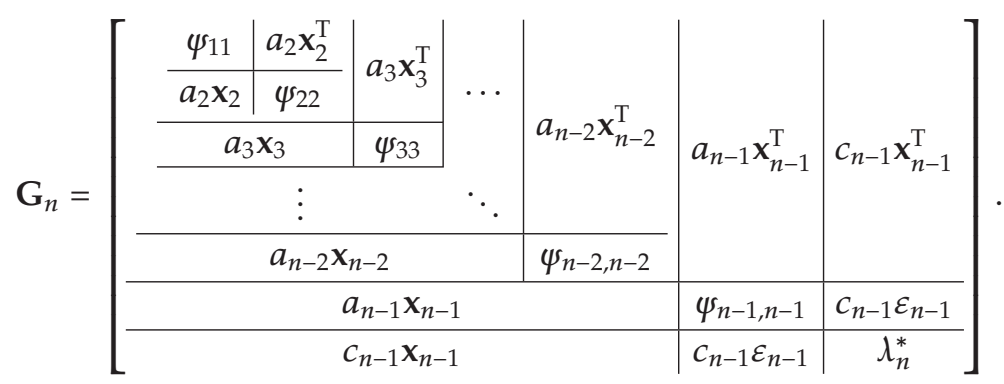

For $k=2, \ldots, n$, we consecutively obtain from $\lambda_{1}^{*}:=\lambda_{1}$ and $\lambda_{k}^{*}:=\lambda_{k-1}^{*} c_{k-1}^{2}+\lambda_{k} d_{k-1}^{2}$ that generally it holds

$$
\lambda_{k}^{*}=\left(c_{1} c_{2} \cdots c_{k-1}\right)^{2} \lambda_{1}+\left(d_{1} c_{2} \cdots c_{k-1}\right)^{2} \lambda_{2}+\cdots+\left(d_{k-2} c_{k-1}\right)^{2} \lambda_{k-1}+d_{k-1}^{2} \lambda_{k}, \quad k=2, \ldots, n .
$$

Since $\psi_{11}=\lambda_{1} a_{1}^{2}+\lambda_{2} b_{1}^{2}$ and $\psi_{k k}:=\lambda_{k}^{*} a_{k}^{2}+\lambda_{k+1} b_{k}^{2}(k=2, \ldots, n-1)$, then from (2.17) it follows that

$$
\begin{aligned}
\psi_{k k}= & \left(c_{1} c_{2} \cdots c_{k-1} a_{k}\right)^{2} \lambda_{1}+\left(d_{1} c_{2} \cdots c_{k-1} a_{k}\right)^{2} \lambda_{2}+\cdots+\left(d_{k-2} c_{k-1} a_{k}\right)^{2} \lambda_{k-1} \\
& +\left(d_{k-1} a_{k}\right)^{2} \lambda_{k}+b_{k}^{2} \lambda_{k+1}, \quad k=2, \ldots, n-1 .
\end{aligned}
$$

Observe that it is not necessary to calculate " $\psi$ "s from (2.18), but only the modified eigenvalues from (2.17) since it holds $\varepsilon_{k}=\left(\lambda_{k}^{*}-\lambda_{k+1}\right) a_{k}$ and $\psi_{k k}=\lambda_{k}^{*} a_{k}^{2}+\lambda_{k+1} b_{k}^{2}=$ 
$\left(\lambda_{k}^{*}-\lambda_{k+1}\right) a_{k}^{2}+\left(a_{k}^{2}+b_{k}^{2}\right) \lambda_{k+1}=a_{k} \varepsilon_{k}+\lambda_{k+1}(k=1, \ldots, n-1)$. As it is $\mathbf{x}_{2}=c_{1} \varepsilon_{1}$, then for $k=2, \ldots, n-2$ from (2.10) it follows that

$$
\begin{aligned}
& \mathbf{x}_{k+1}=c_{k}\left[\mathbf{x}_{k} \mid \varepsilon_{k}\right] \\
& =c_{k}\left[c_{k-1}\left[\mathbf{x}_{k-1} \mid \varepsilon_{k-1}\right] \mid \varepsilon_{k}\right] \\
& =\left[\begin{array}{l|l|l|l}
c_{k} c_{k-1} \mathbf{x}_{k-1} & \mid c_{k} c_{k-1} \varepsilon_{k-1} & c_{k} \varepsilon_{k}
\end{array}\right] \\
& =\cdots=\left[c_{k} c_{k-1} \cdots c_{2} \mathbf{x}_{2}\left|c_{k} c_{k-1} \cdots c_{2} \varepsilon_{2}\right| \cdots\left|c_{k} c_{k-1} \varepsilon_{k-1}\right| c_{k} \varepsilon_{k}\right] \\
& =\left[c_{k} c_{k-1} \cdots c_{2} c_{1} \varepsilon_{1}\left|c_{k} c_{k-1} \cdots c_{2} \varepsilon_{2}\right| \cdots\left|c_{k} c_{k-1} \varepsilon_{k-1}\right| c_{k} \varepsilon_{k}\right] \\
& a_{k} \mathbf{x}_{k}=\left[a_{k} c_{k-1} c_{k-2} \cdots c_{2} c_{1} \varepsilon_{1}\left|a_{k} c_{k-1} c_{k-2} \cdots c_{2} \varepsilon_{2}\right| \cdots\left|a_{k} c_{k-1} c_{k-2} \varepsilon_{k-2}\right| a_{k} c_{k-1} \varepsilon_{k-1}\right] \text {, } \\
& k=2, \ldots, n-1 \text {. }
\end{aligned}
$$

The real symmetric matrix $\mathbf{G}_{n}$ with assigned spectrum $\left\{\lambda_{1}, \lambda_{2}, \ldots, \lambda_{n}\right\}$ and the explicitly expressed entries can be derived from (2.16) and (2.20), bearing on mind that " $\psi$ "s and " $\varepsilon^{\prime \prime}$ s are calculated by using $\left\{\lambda_{1}, \lambda_{2}, \ldots, \lambda_{n}\right\}, \mathbf{P}_{k}$, modified eigenvalues (2.17) and $\varepsilon_{k}=\left(\lambda_{k}^{*}-\right.$ $\left.\lambda_{k+1}\right) a_{k}(k=1, \ldots, n-1)$ :

$\mathbf{G}_{n}=\left[\begin{array}{c|c|c|c|c|c|c}\psi_{11} & a_{2} c_{1} \varepsilon_{1} & a_{3} c_{2} c_{1} \varepsilon_{1} & a_{4} c_{3} c_{2} c_{1} \varepsilon_{1} & \cdots & p & \mathcal{S} \\ \hline a_{2} c_{1} \varepsilon_{1} & \psi_{22} & a_{3} c_{2} \varepsilon_{2} & a_{4} c_{3} c_{2} \varepsilon_{2} & \cdots & \mathcal{U} & \boldsymbol{f} \\ \hline a_{3} c_{2} c_{1} \varepsilon_{1} & a_{3} c_{2} \varepsilon_{2} & \vdots & \vdots & \cdots & \vdots & \vdots \\ \hline a_{4} c_{3} c_{2} c_{1} \varepsilon_{1} & a_{4} c_{3} c_{2} \varepsilon_{2} & \vdots & \vdots & \vdots & \vdots & \vdots \\ \hline \vdots & \vdots & \vdots & \vdots & \ddots & \vdots & \vdots \\ \hline D & \mathcal{U} & \cdots & \cdots & \cdots & \psi_{n-1, n-1} & c_{n-1} \varepsilon_{n-1} \\ \hline \mathcal{S} & \boldsymbol{f} & \cdots & \cdots & \cdots & c_{n-1} \varepsilon_{n-1} & \lambda_{n}^{*}\end{array}\right]$,

where $\boldsymbol{P}$ denotes $a_{n-1} c_{n-2} \cdots c_{2} c_{1} \varepsilon_{1}, \mathcal{S}$ denotes $c_{n-1} c_{n-2} \cdots c_{2} c_{1} \varepsilon_{1}, \boldsymbol{U}$ denotes $a_{n-1} c_{n-2} \cdots c_{2} \varepsilon_{2}$, and $\mp$ denotes $c_{n-1} c_{n-2} \cdots c_{2} \varepsilon_{2}$. Entries of $\mathbf{G}_{n}=\mathbf{G}_{n}^{\mathrm{T}}=\left[g_{k m}\right]_{n \times n}$ are $g_{k k}=\psi_{k k}(k=1, \ldots, n-1)$, $g_{n n}=\lambda_{n}^{*}, g_{k m}=a_{k} c_{k-1} c_{k-2} \cdots c_{m} \varepsilon_{m}(k>m ; k=2, \ldots, n-1)$ and $g_{n m}=c_{n-1} c_{n-2} \cdots c_{m} \varepsilon_{m}(m=$ $1, \ldots, n-1)$. They are calculated according to the following steps:

(a) Select arbitrarily the entries $\left\{a_{k}, b_{k}, c_{k}, d_{k}\right\}$ of $2 \times 2$ orthogonal matrices $\mathbf{P}_{k}(k=$ $1, \ldots, n-1)$, given by $(2.1)$;

(b) with $\lambda_{1}^{*}:=\lambda_{1}$, calculate the modified eigenvalues $\lambda_{k}^{*}(k=2, \ldots, n)$, by using $(2.17)$;

(c) calculate $\varepsilon_{k}=\left(\lambda_{k}^{*}-\lambda_{k+1}\right) a_{k}$ and $\psi_{k k}=a_{k} \varepsilon_{k}+\lambda_{k+1}(k=1, \ldots, n-1)$;

(d) calculate the entries of $\mathbf{G}_{n}$, by using (2.21).

Matrix $\mathbf{U}(2.4)$ is $n \times n$ orthogonal modal matrix established from eigenvectors of $\mathbf{G}_{n}$. We will now prove that $\mathbf{U}$ is not only orthogonal, but also lower Hessenberg with explicitly 
expressed entries. Let us firstly produce $\mathbf{U}_{1}^{\mathrm{T}} \mathbf{U}_{2}^{\mathrm{T}}$ and $\mathbf{U}_{1}^{\mathrm{T}} \mathbf{U}_{2}^{\mathrm{T}} \mathbf{U}_{3}^{\mathrm{T}}$, whose partition will enable us to anticipate the partition of $\mathbf{U}_{1}^{\mathrm{T}} \mathbf{U}_{2}^{\mathrm{T}} \mathbf{U}_{3}^{\mathrm{T}} \cdots \mathbf{U}_{k}^{\mathrm{T}}(k=4, \ldots, n-1)$

$$
\mathbf{U}_{1}^{\mathrm{T}} \mathbf{U}_{2}^{\mathrm{T}}=\left[\begin{array}{ccc|c}
a_{1} & a_{2} c_{1} & c_{2} c_{1} & \\
b_{1} & a_{2} d_{1} & c_{2} d_{1} & \mathbf{0}_{3, n-3} \\
0 & b_{2} & d_{2} & \\
\hline & \mathbf{0}_{3, n-3}^{\mathrm{T}} & \mathbf{I}_{n-3}
\end{array}\right], \quad \mathbf{U}_{1}^{\mathrm{T}} \mathbf{U}_{2}^{\mathrm{T}} \mathbf{U}_{3}^{\mathrm{T}}=\left[\begin{array}{cccc|c}
a_{1} & a_{2} c_{1} & a_{3} c_{2} c_{1} & c_{3} c_{2} c_{1} & \\
b_{1} & a_{2} d_{1} & a_{3} c_{2} d_{1} & c_{3} c_{2} d_{1} & \mathbf{0}_{4, n-4} \\
0 & b_{2} & a_{3} d_{2} & c_{3} d_{2} & \\
0 & 0 & b_{3} & d_{3} & \\
\hline & & \mathbf{0}_{4, n-4}^{\mathrm{T}} & & \mathbf{I}_{n-4}
\end{array}\right] .
$$

If we now suppose that $\mathbf{U}_{1}^{\mathrm{T}} \mathbf{U}_{2}^{\mathrm{T}} \cdots \mathbf{U}_{k}^{\mathrm{T}}:=\mathbf{H}_{k+1} \oplus \mathbf{I}_{n-k-1}(k=2, \ldots, n-1)$, where $\mathbf{H}_{k+1}$ is orthogonal $(k+1) \times(k+1)$ upper Hessenberg matrix

$$
\mathbf{H}_{k+1}=\left[\begin{array}{c|c|c|c|c|c}
a_{1} & a_{2} c_{1} & a_{3} c_{2} c_{1} & \cdots & a_{k} c_{k-1} \cdots c_{2} c_{1} & c_{k} c_{k-1} \cdots c_{2} c_{1} \\
\hline b_{1} & a_{2} d_{1} & a_{3} c_{2} d_{1} & \cdots & a_{k} c_{k-1} \cdots c_{2} d_{1} & c_{k} c_{k-1} \cdots c_{2} d_{1} \\
\hline 0 & b_{2} & a_{3} d_{2} & \cdots & a_{k} c_{k-1} \cdots c_{3} d_{2} & c_{k} c_{k-1} \cdots c_{3} d_{2} \\
\hline \cdots & \cdots & \cdots & \cdots & \cdots & \cdots \\
\hline 0 & \cdots & 0 & b_{k-1} & a_{k} d_{k-1} & c_{k} d_{k-1} \\
\hline 0 & \cdots & \cdots & 0 & b_{k} & d_{k}
\end{array}\right],
$$

then since according to (2.2), it holds $\mathbf{U}_{k+1}:=\mathbf{I}_{k} \oplus \mathbf{P}_{k+1} \oplus \mathbf{I}_{n-k-2}$, we may write further for $k=2, \ldots, n-2$

$$
\begin{aligned}
\mathbf{U}_{1}^{\mathrm{T}} \mathbf{U}_{2}^{\mathrm{T}} \cdots \mathbf{U}_{k}^{\mathrm{T}} \mathbf{U}_{k+1}^{\mathrm{T}} & =\left(\mathbf{H}_{k+1} \oplus \mathbf{I}_{n-k-1}\right) \mathbf{U}_{k+1}^{\mathrm{T}} \\
& =\left(\mathbf{H}_{k+1} \oplus 1 \oplus \mathbf{I}_{n-k-2}\right)\left(\mathbf{I}_{k} \oplus \mathbf{P}_{k+1}^{\mathrm{T}} \oplus \mathbf{I}_{n-k-2}\right) \\
& =\left[\left(\mathbf{H}_{k+1} \oplus 1\right)\left(\mathbf{I}_{k} \oplus \mathbf{P}_{k+1}^{\mathrm{T}}\right)\right] \oplus \mathbf{I}_{n-k-2} \\
& =\mathbf{H}_{k+2} \oplus \mathbf{I}_{n-k-2}, \quad \text { where } \mathbf{H}_{k+2}:=\left(\mathbf{H}_{k+1} \oplus 1\right)\left(\mathbf{I}_{k} \oplus \mathbf{P}_{k+1}^{\mathrm{T}}\right) .
\end{aligned}
$$

By using (2.2), (2.23)-(2.24), it follows that

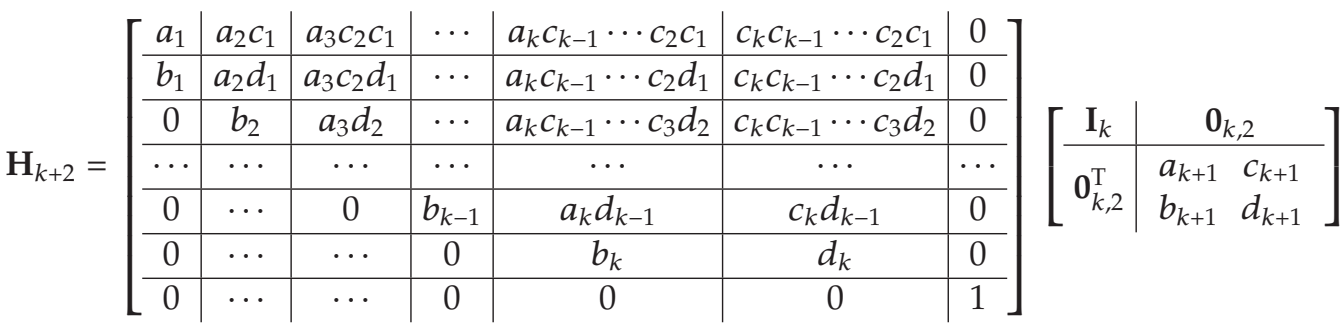

$$
\begin{aligned}
& =\left[\begin{array}{c|c|c|c|c|c|c}
a_{1} & a_{2} c_{1} & a_{3} c_{2} c_{1} & \cdots & a_{k} c_{k-1} \cdots c_{2} c_{1} & a_{k+1} c_{k} c_{k-1} \cdots c_{2} c_{1} & c_{k+1} c_{k} c_{k-1} \cdots c_{2} c_{1} \\
\hline b_{1} & a_{2} d_{1} & a_{3} c_{2} d_{1} & \cdots & a_{k} c_{k-1} \cdots c_{2} d_{1} & a_{k+1} c_{k} c_{k-1} \cdots c_{2} d_{1} & c_{k+1} c_{k} c_{k-1} \cdots c_{2} d_{1} \\
\hline 0 & b_{2} & a_{3} d_{2} & \cdots & a_{k} c_{k-1} \cdots c_{3} d_{2} & a_{k+1} c_{k} c_{k-1} \cdots c_{3} d_{2} & c_{k+1} c_{k} c_{k-1} \cdots c_{3} d_{2} \\
\hline \cdots & \cdots & \cdots & \cdots & \cdots & \cdots & \cdots \\
\hline 0 & \cdots & 0 & b_{k-1} & a_{k} d_{k-1} & a_{k+1} c_{k} d_{k-1} & c_{k+1} c_{k} d_{k-1} \\
\hline 0 & \cdots & \cdots & 0 & b_{k} & a_{k+1} d_{k} & c_{k+1} d_{k} \\
\hline 0 & \cdots & \cdots & 0 & 0 & b_{k+1} & d_{k+1}
\end{array}\right],
\end{aligned}
$$


and thereby it is proved our previous assumption that $\mathbf{U}_{1}^{\mathrm{T}} \mathbf{U}_{2}^{\mathrm{T}} \cdots \mathbf{U}_{k}^{\mathrm{T}}:=\mathbf{H}_{k+1} \oplus \mathbf{I}_{n-k-1}(k=$ $2, \ldots, n-1)$, where $\mathbf{H}_{k+1}(2.23)$ is the orthogonal upper Hessenberg $(k+1) \times(k+1)$ matrix with entries expressed explicitly. And finally, for $k=n-1$ from $\mathbf{U}_{1}^{\mathrm{T}} \mathbf{U}_{2}^{\mathrm{T}} \cdots \mathbf{U}_{k}^{\mathrm{T}}:=\mathbf{H}_{k+1} \oplus \mathbf{I}_{n-k-1}$ and (2.4), (2.23), we obtain $\mathbf{H}_{n}=\mathbf{U}^{\mathrm{T}}=\mathbf{U}_{1}^{\mathrm{T}} \mathbf{U}_{2}^{\mathrm{T}} \cdots \mathbf{U}_{n-1}^{\mathrm{T}}$ and

$\mathbf{U}=\mathbf{H}_{n}^{\mathrm{T}}=\mathbf{U}_{n-1} \mathbf{U}_{n-2} \cdots \mathbf{U}_{1}=\left[\begin{array}{c|c|c|c|c|c|c}a_{1} & b_{1} & 0 & 0 & \cdots & 0 & 0 \\ \hline a_{2} c_{1} & a_{2} d_{1} & b_{2} & 0 & \cdots & 0 & 0 \\ \hline a_{3} c_{2} c_{1} & a_{3} c_{2} d_{1} & a_{3} d_{2} & \vdots & \cdots & \vdots & \vdots \\ \hline a_{4} c_{3} c_{2} c_{1} & a_{4} c_{3} c_{2} d_{1} & a_{4} c_{3} d_{2} & \vdots & \vdots & \vdots & \vdots \\ \hline \vdots & \vdots & \vdots & \vdots & \ddots & \vdots & \vdots \\ \hline a_{n-1} c_{n-2} \cdots c_{2} c_{1} & a_{n-1} c_{n-2} \cdots c_{2} d_{1} & \cdots & \cdots & \cdots & a_{n-1} d_{n-2} & b_{n-1} \\ \hline c_{n-1} c_{n-2} \cdots c_{2} c_{1} & c_{n-1} c_{n-2} \cdots c_{2} d_{1} & \cdots & \cdots & \cdots & c_{n-1} d_{n-2} & d_{n-1}\end{array}\right]$.

The entries of the orthogonal lower Hessenberg matrix $\mathbf{U}=\left[u_{k m}\right](k, m=1, \ldots, n)$ are defined as follows:

$$
\begin{gathered}
u_{k m}=0 \quad(m>k+1 ; k, m=1, \ldots, n), \quad u_{k, k+1}=b_{k} \quad(k=1, \ldots, n-1), \quad u_{11}=a_{1}, \\
u_{k k}=a_{k} d_{k-1} \quad(k=2, \ldots, n-1), \quad u_{k, 1}=a_{k} c_{k-1} c_{k-2} \cdots c_{2} c_{1} \quad(k=2, \ldots, n-1), \\
u_{n, 1}=c_{n-1} c_{n-2} \cdots c_{1}, \quad u_{n, k}=c_{n-1} c_{n-2} \cdots c_{k} d_{k-1} \quad(k=2, \ldots, n-1), \\
u_{k m}=a_{k} c_{k-1} \cdots c_{m} d_{m-1} \quad(m+1 \leq k \leq n-1 ; m=2, \ldots, n-1) .
\end{gathered}
$$

By using the similar arguments as in derivation of entries of matrix $\mathbf{U}$, the orthogonal matrix V which is to be produced by using (2.4) can be shown to take on upper Hessenberg form. Proving of this fact goeswith similar paces that were used for obtaining $\mathbf{U}$ and it is left to the reader.

\section{The explicit solution of the IEP of real symmetric matrices with some specific sign patterns}

Let the real eigenvalues from the spectrum $\left\{\lambda_{1}, \lambda_{2}, \ldots, \lambda_{n}\right\}$ be arbitrarily enumerated, thereby establishing the sequence $\left\{\lambda_{k}\right\}(k=1, \ldots, n)$. The nonnegative sequence will be denoted by $\left\{\lambda_{k}\right\} \geq 0$, and the nonpositive one by $\left\{\lambda_{k}\right\} \leq 0(k=1, \ldots, n)$. Firstly, we will prove two lemmas.

Lemma 3.1. If the sequence $\left\{\lambda_{k}\right\} \geq 0(k=1, \ldots, n)$ is increasing [decreasing], then in (2.21) $\lambda_{n}^{*} \geq$ $0, \psi_{m m} \geq 0$, and the sequence $\left\{a_{m} \varepsilon_{m}\right\} \leq 0\left[\left\{a_{m} \varepsilon_{m}\right\} \geq 0\right](m=1, \ldots, n-1)$.

Proof. Since $\left\{\lambda_{k}\right\} \geq 0(k=1, \ldots, n)$, then it is trivial to see from (2.17) and (2.18) that all diagonal entries of $\mathbf{G}_{n}$ are nonnegative, that is, $\lambda_{n}^{*} \geq 0$ and $\psi_{m m} \geq 0(m=1, \ldots, n-1)$ no matter whether the sequence $\left\{\lambda_{k}\right\} \geq 0$ is increasing or decreasing. By virtue of orthogonality 
of $\mathbf{P}_{k}$, we have $c_{k}^{2}+d_{k}^{2}=1(k=1, \ldots, n)$. If $\left\{\lambda_{k}\right\}(k=1, \ldots, n)$ is increasing sequence, then for $m=1$ we have $a_{1} \varepsilon_{1}=\left(\lambda_{1}^{*}-\lambda_{2}\right) a_{1}^{2}=\left(\lambda_{1}-\lambda_{2}\right) a_{1}^{2} \leq 0$ and for $m=2, \ldots, n-1$ we obtain

$$
\begin{aligned}
& d_{m-1}^{2} \lambda_{m}-\lambda_{m+1} \\
& \quad \leq d_{m-1}^{2} \lambda_{m}-\lambda_{m}=\lambda_{m}\left(1-d_{m-1}^{2}\right)=-c_{m-1}^{2} \lambda_{m} \leq-c_{m-1}^{2} \lambda_{m-1} \\
& \left(d_{m-2} c_{m-1}\right)^{2} \lambda_{m-1}+d_{m-1}^{2} \lambda_{m}-\lambda_{m+1} \\
& \quad \leq\left(d_{m-2} c_{m-1}\right)^{2} \lambda_{m-1}-c_{m-1}^{2} \lambda_{m-1}=-\left(c_{m-2} c_{m-1}\right)^{2} \lambda_{m-1} \leq-\left(c_{m-2} c_{m-1}\right)^{2} \lambda_{m-2} \\
& \left(d_{m-3} c_{m-2} c_{m-1}\right)^{2} \lambda_{m-2}+\left(d_{m-2} c_{m-1}\right)^{2} \lambda_{m-1}+d_{m-1}^{2} \lambda_{m}-\lambda_{m+1} \\
& \quad \leq-\left(c_{m-3} c_{m-2} c_{m-1}\right)^{2} \lambda_{m-2} \leq-\left(c_{m-3} c_{m-2} c_{m-1}\right)^{2} \lambda_{m-3} \\
& \left(d_{1} c_{2} \cdots c_{m-1}\right)^{2} \lambda_{2}+\cdots+\left(d_{m-2} c_{m-1}\right)^{2} \lambda_{m-1}+d_{m-1}^{2} \lambda_{m}-\lambda_{m+1} \\
& \quad \leq-\left(c_{1} \cdots c_{m-1}\right)^{2} \lambda_{2} \leq-\left(c_{1} \cdots c_{m-1}\right)^{2} \lambda_{1} .
\end{aligned}
$$

From (2.17) and the last of inequalities (3.1) it follows $\lambda_{m}^{*} \leq \lambda_{m+1}$ and $a_{m} \varepsilon_{m}=\left(\lambda_{m}^{*}-\lambda_{m+1}\right) a_{m}^{2} \leq$ $0(m=2, \ldots, n-1)$. If $\left\{\lambda_{k}\right\}(k=1, \ldots, n)$ is decreasing sequence, then for $m=1$ we have $a_{1} \varepsilon_{1}=\left(\lambda_{1}^{*}-\lambda_{2}\right) a_{1}^{2}=\left(\lambda_{1}-\lambda_{2}\right) a_{1}^{2} \geq 0$ and for $m=2, \ldots, n-1$ we obtain

$$
\begin{aligned}
& d_{m-1}^{2} \lambda_{m}-\lambda_{m+1} \\
& \quad \geq d_{m-1}^{2} \lambda_{m}-\lambda_{m}=-\lambda_{m}\left(1-d_{m-1}^{2}\right)=-c_{m-1}^{2} \geq-c_{m-1}^{2} \lambda_{m-1} \\
& \left(d_{m-2} c_{m-1}\right)^{2} \lambda_{m-1}+d_{m-1}^{2} \lambda_{m}-\lambda_{m+1} \\
& \quad \geq\left(d_{m-2} c_{m-1}\right)^{2} \lambda_{m-1}-c_{m-1}^{2} \lambda_{m-1}=-\left(c_{m-2} c_{m-1}\right)^{2} \lambda_{m-1} \geq-\left(c_{m-2} c_{m-1}\right)^{2} \lambda_{m-2} \\
& \left(d_{m-3} c_{m-2} c_{m-1}\right)^{2} \lambda_{m-2}+\left(d_{m-2} c_{m-1}\right)^{2} \lambda_{m-1}+d_{m-1}^{2} \lambda_{m}-\lambda_{m+1} \\
& \quad \geq-\left(c_{m-3} c_{m-2} c_{m-1}\right)^{2} \lambda_{m-2} \geq-\left(c_{m-3} c_{m-2} c_{m-1}\right)^{2} \lambda_{m-3} \\
& \left(d_{1} c_{2} \cdots c_{m-1}\right)^{2} \lambda_{2}+\cdots+\left(d_{m-2} c_{m-1}\right)^{2} \lambda_{m-1}+d_{m-1}^{2} \lambda_{m}-\lambda_{m+1} \\
& \geq-\left(c_{1} \cdots c_{m-1}\right)^{2} \lambda_{2} \geq-\left(c_{1} \cdots c_{m-1}\right)^{2} \lambda_{1} .
\end{aligned}
$$

From (2.17) and the last of inequalities (3.2) it follows $\lambda_{m}^{*} \geq \lambda_{m+1}$ and $a_{m} \varepsilon_{m}=\left(\lambda_{m}^{*}-\lambda_{m+1}\right) a_{m}^{2} \geq$ $0(m=2, \ldots, n-1)$. This completes the proof of lemma. For a nonpositive sequence, an analogous lemma can be formulated. 
Lemma 3.2. If the sequence $\left\{\lambda_{k}\right\} \leq 0(k=1, \ldots, n)$ is increasing [decreasing], then in (2.21) $\lambda_{n}^{*} \leq$ $0, \psi_{m m} \leq 0$ and the sequence $\left\{a_{m} \varepsilon_{m}\right\} \leq 0\left[\left\{a_{m} \varepsilon_{m}\right\} \geq 0\right](m=1, \ldots, n-1)$.

Proof. It is similar to that of Lemma 3.1, but in this case the diagonal entries of $\mathbf{G}_{n}$ are nonpositive, that is, $\lambda_{n}^{*} \leq 0$ and $\psi_{m m} \leq 0(m=1, \ldots, n-1)$, no matter whether the sequence $\left\{\lambda_{k}\right\} \leq 0$ is increasing or decreasing (see (2.18)).

Now, we shall formulate a new theorem related to explicit solving of IEP of real symmetric matrices with some specific sign patterns.

Theorem 3.3. If $\theta_{k}(k=1, \ldots, n-1)$ are arbitrarily selected angles from the range $[0, \pi / 2]$, then the entries of real symmetric matrices $\mathbf{G}_{n}$ with assigned spectrum $\left\{\lambda_{1}, \lambda_{2}, \ldots, \lambda_{n}\right\}$, produced by (2.21), can attain the following twelve sign patterns (zero entries are permitted), depending on selection of matrices $\mathbf{P}_{k}(k=1, \ldots, n-1)$ (see (2.1)).

Case 1.

$$
\begin{aligned}
& \mathbf{P}_{k}=\mathbf{A}_{k}=\left[\begin{array}{cc}
\cos \theta_{k} & -\sin \theta_{k} \\
\sin \theta_{k} & \cos \theta_{k}
\end{array}\right], \quad \text { or } \quad \mathbf{P}_{k}=\mathbf{B}_{k}=\left[\begin{array}{cc}
\cos \theta_{k} & \sin \theta_{k} \\
\sin \theta_{k} & -\cos \theta_{k}
\end{array}\right] \Longrightarrow \\
& \mathbf{G}_{n}=\left[\begin{array}{cccc}
+ & & & \\
& + & & + \\
& \ddots & \\
+ & & \\
& & & +
\end{array}\right], \quad \mathbf{G}_{n}=\left[\begin{array}{cccc}
- & & & \\
- & & + \\
& \ddots & \\
+ & & \\
& & & -
\end{array}\right] \text {, } \\
& \lambda_{1} \geq \lambda_{2} \geq \cdots \geq \lambda_{n} \geq 0 \quad \lambda_{n} \leq \lambda_{n-1} \leq \cdots \leq \lambda_{1} \leq 0 \\
& \mathbf{G}_{n}=\left[\begin{array}{cccc}
+ & & & \\
+ & & - \\
& \ddots & \\
- & & \\
& & & +
\end{array}\right], \quad \mathbf{G}_{n}=\left[\begin{array}{cccc}
- & & \\
- & & - \\
& \ddots & \\
- & & \\
& & & -
\end{array}\right] \text {. } \\
& \lambda_{n} \geq \lambda_{n-1} \geq \cdots \geq \lambda_{1} \geq 0 \quad \lambda_{1} \leq \lambda_{2} \leq \cdots \leq \lambda_{n} \leq 0
\end{aligned}
$$

Case 2.

$$
\begin{gathered}
\mathbf{P}_{k}=\mathbf{C}_{k}=\left[\begin{array}{ccc}
\cos \theta_{k} & \sin \theta_{k} \\
-\sin \theta_{k} & \cos \theta_{k}
\end{array}\right] \Longrightarrow \\
\mathbf{G}_{n}=\left[\begin{array}{ccccc}
+ & - & + & \cdots & (-1)^{n-1} \\
- & + & - & \cdots & (-1)^{n-2} \\
\cdots & \cdots & \cdots & \cdots & \cdots \\
(-1)^{n-2} & \cdots & \cdots & + & - \\
(-1)^{n-1} & \cdots & \cdots & - & +
\end{array}\right], \quad \mathbf{G}_{n}=\left[\begin{array}{ccccc}
- & - & + & \cdots & (-1)^{n-1} \\
- & - & - & \cdots & (-1)^{n-2} \\
\cdots & \cdots & \cdots & \cdots & \cdots \\
(-1)^{n-2} & \cdots & \cdots & - & - \\
(-1)^{n-1} & \cdots & \cdots & - & -
\end{array}\right], \\
\lambda_{1} \geq \lambda_{2} \geq \cdots \geq \lambda_{n} \geq 0 \\
\lambda_{n} \leq \lambda_{n-1} \leq \cdots \leq \lambda_{1} \leq 0
\end{gathered}
$$




$$
\begin{gathered}
\mathbf{G}_{n}=\left[\begin{array}{ccccc}
+ & + & - & \cdots & (-1)^{n} \\
+ & + & + & \cdots & (-1)^{n-1} \\
\cdots & \cdots & \cdots & \cdots & \cdots \\
(-1)^{n-1} & \cdots & \cdots & + & + \\
(-1)^{n} & \cdots & \cdots & + & +
\end{array}\right], \quad \mathbf{G}_{n}=\left[\begin{array}{ccccc}
- & + & - & \cdots & (-1)^{n} \\
+ & - & + & \cdots & (-1)^{n-1} \\
\cdots & \cdots & \cdots & \cdots & \cdots \\
(-1)^{n-1} & \cdots & \cdots & - & + \\
(-1)^{n} & \cdots & \cdots & + & -
\end{array}\right] . \\
\lambda_{n} \geq \lambda_{n-1} \geq \cdots \geq \lambda_{1} \geq 0 \\
\lambda_{1} \leq \lambda_{2} \leq \cdots \leq \lambda_{n} \leq 0
\end{gathered}
$$

Case 3.

$$
\begin{aligned}
& \mathbf{P}_{k}=\mathbf{D}_{k}=\left[\begin{array}{cc}
-\cos \theta_{k} & \sin \theta_{k} \\
\sin \theta_{k} & \cos \theta_{k}
\end{array}\right] \Longrightarrow \\
& \mathbf{G}_{n}=\left[\begin{array}{cccccc}
+ & + & + & \cdots & + & - \\
+ & + & + & \cdots & + & - \\
\cdots & \cdots & \cdots & \cdots & \cdots & \cdots \\
+ & + & + & \cdots & + & - \\
- & - & - & \cdots & - & +
\end{array}\right], \quad \mathbf{G}_{n}=\left[\begin{array}{cccccc}
- & + & + & \cdots & + & - \\
+ & - & + & \cdots & + & - \\
\cdots & \cdots & \cdots & \cdots & \cdots & \cdots \\
+ & + & \cdots & \cdots & - & - \\
- & - & \cdots & \cdots & - & -
\end{array}\right], \\
& \lambda_{1} \geq \lambda_{2} \geq \cdots \geq \lambda_{n} \geq 0 \quad \lambda_{n} \leq \lambda_{n-1} \leq \cdots \leq \lambda_{1} \leq 0 \\
& \mathbf{G}_{n}=\left[\begin{array}{cccccc}
+ & - & - & \cdots & - & + \\
- & + & - & \cdots & - & + \\
\cdots & \cdots & \cdots & \cdots & \cdots & \cdots \\
- & - & \cdots & \cdots & + & + \\
+ & + & \cdots & \cdots & + & +
\end{array}\right], \quad \mathbf{G}_{n}=\left[\begin{array}{cccccc}
- & - & - & \cdots & - & + \\
- & - & - & \cdots & - & + \\
\cdots & \cdots & \cdots & \cdots & \cdots & \cdots \\
- & - & \cdots & \cdots & - & + \\
+ & + & \cdots & \cdots & + & +
\end{array}\right] \text {. } \\
& \lambda_{n} \geq \lambda_{n-1} \geq \cdots \geq \lambda_{1} \geq 0 \quad \lambda_{1} \leq \lambda_{2} \leq \cdots \leq \lambda_{n} \leq 0
\end{aligned}
$$

Proof. If $\theta_{k} \in[0, \pi / 2]$, then the signs of $a_{k}$ and $c_{k}$ depend solely on selection of canonic orthogonal matrices $\mathbf{P}_{k}(k=1, \ldots, n-1)$. For any sign of sequence $\left\{\lambda_{m}\right\}(m=1, \ldots, n)$ and its monotonicy realized through enumeration of its members, one can readily check the sign patterns stated above: by using (2.18) to determine signs of the diagonal entries in $\mathbf{G}_{n}$ and by using Lemma 3.1 or Lemma 3.2 to determine signs of $\varepsilon_{k}(k=1, \ldots, n-1)$. Observe that only in Case 1 when $\lambda_{n} \geq \lambda_{n-1} \geq \cdots \geq \lambda_{1} \geq 0$, that is, when the sequence $\left\{\lambda_{m}\right\}(m=1, \ldots, n)$ is nonnegative and increasing (but not strictly), matrix $\mathbf{G}_{n}$ is produced with hd sign pattern, including the possible presence of zero entries. $\mathbf{G}_{n}$ may attain a sparse structure if, for example, some eigenvalues are equal. To see that, let us firstly suppose $\lambda_{1}=\cdots=\lambda_{k}=\lambda$. Then from (2.17)-(2.18) it follows that $\lambda_{1}^{*}=\cdots=\lambda_{k}^{*}=\lambda, \psi_{11}=\cdots=\psi_{k-1, k-1}=\lambda$ and $\varepsilon_{1}=\cdots=$ $\varepsilon_{k-1}=0$, thus obviously making the matrix $\mathbf{G}_{n}$ (2.21) with sparse structure. By using (2.17)(2.18), (2.21) and both Lemmas, we can readily infer that if $\theta_{k} \in(0, \pi / 2)(k=1, \ldots, n-1)$ and the sequence $\left\{\lambda_{m}\right\}(m=1, \ldots, n)$ is strictly monotone, then matrix $\mathbf{G}_{n}(2.21)$ is produced with no zero entries in all three considered cases.

Remark 3.4. Let $\lambda_{1} \lambda_{2} \cdots \lambda_{n} \neq 0$. Then, since $\mathbf{G}_{n}=\mathbf{U G}_{1} \mathbf{U}^{\mathrm{T}}$ and $\mathbf{U}^{-1}=\mathbf{U}^{\mathrm{T}}$ (recall that $\mathbf{U}$ is orthogonal), it follows that $\mathbf{G}_{n}^{-1}=\left(\mathbf{U}^{\mathrm{T}}\right)^{-1} \mathbf{G}_{1}^{-1} \mathbf{U}^{-1}=\mathbf{U G}_{1}^{-1} \mathbf{U}^{\mathrm{T}}$. Also, when the sequence $\left\{\lambda_{m}\right\}(m=1, \ldots, n)$ is increasing (decreasing), then the sequence $\left\{\lambda_{m}^{-1}\right\}(m=1, \ldots, n)$ is decreasing (increasing). These facts and Theorem 3.3 offer a possibility of determining the 
sign pattern of $\mathbf{G}_{n}^{-1}$ without really inverting $\mathbf{G}_{n}$. Furthermore, by using (2.17)-(2.18), (2.21), $\mathbf{G}_{n}^{-1}$ can be calculated explicitly, also without really inverting $\mathbf{G}_{n}$.

Theorem 3.5. Let the positive increasing sequence $\left\{\lambda_{m}\right\}(m=1, \ldots, n)$ be the spectrum of $\mathbf{G}_{n}$ produced by using Case 1 of Theorem 3.3. Then there always exists such a diagonal matrix $\mathbf{D}:=$ $\operatorname{diag}\left(d_{1}, d_{2}, \ldots, d_{n}\right)$ with positive diagonal entries which makes $\mathbf{D G}_{n} \mathbf{D}$ truly hyperdominant.

Proof. If $\mathrm{G}_{1}:=\operatorname{diag}\left(\lambda_{1}, \lambda_{2}, \ldots, \lambda_{n}\right)$, then by Case 1 of Theorem 3.3, the nonsingular matrix $\mathbf{G}_{n}=\mathbf{U G}_{1} \mathbf{U}^{\mathrm{T}}$ will have hd sign pattern and by Remark $3.4 \mathbf{G}_{n}^{-1}=\mathbf{U G}_{1}^{-1} \mathbf{U}^{\mathrm{T}}$ will be nonnegative matrix. Since $d_{m}>0(m=1, \ldots, n)$, then the nonsingular symmetric matrix $\mathbf{D G}_{n} \mathbf{D}$ is produced with hd sign pattern, but it may not be truly hd, unless hd margin of each of its rows (or columns) is nonnegative (recall that hd margin of a row or a column is sum of all entries in that row or column). If $\mathbf{G}_{n}=\left[g_{k m}\right](k, m=1, \ldots, n)$, then hd margin $p_{k}$ of the $k$ th row (or the $k$ th column) in $\mathbf{D G} \mathbf{G}_{n} \mathbf{D}$ is given by

$$
p_{k}=\sum_{m=1}^{n} g_{k m} d_{m} d_{k}=d_{k} \alpha_{k}, \quad \text { where } \alpha_{k}:=\sum_{m=1}^{n} g_{k m} d_{m}, k=1, \ldots, n .
$$

Let we arbitrarily select $\alpha_{k}>0(k=1, \ldots, n)$ and let a := $\left[\begin{array}{lll}\alpha_{1} & \alpha_{2} \cdots \alpha_{n}\end{array}\right]^{\mathrm{T}}, \operatorname{col}(\mathbf{D}):=$

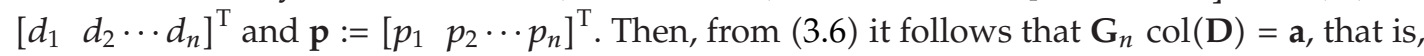
$\operatorname{col}(\mathbf{D})=\mathbf{G}_{n}^{-1} \mathbf{a}>\mathbf{0}_{n, 1}$ and $\mathbf{p}=\mathbf{D a}>\mathbf{0}_{n, 1}$. This not only means that $\mathbf{D G}_{n} \mathbf{D}$ has hd sign pattern, but that it is truly hd furthermore. Obviously, as much as " $\alpha$ "s are assumed greater, the greater will be row (column) hd margins of $\mathbf{D} \mathbf{G}_{n} \mathbf{D}$. This completes the proof of theorem.

\section{Explicit solution of IEP of hd matrices with uncommitted and with assigned nonnegative spectrum}

Theorem 4.1. Let $\theta_{k}(k=1, \ldots, n-1)$ be a set of angles selected from the range $[0, \pi / 2]$ and let $\left\{\lambda_{1}, \lambda_{2}, \ldots, \lambda_{n}\right\}$ be uncommitted nonnegative spectrum of the real symmetric matrix $\mathbf{G}_{n}=$ $\mathbf{U G}_{1} \mathbf{U}^{\mathrm{T}}\left[\mathbf{G}_{1}=\operatorname{diag}\left(\lambda_{1}, \lambda_{2}, \ldots, \lambda_{n}\right)\right]$ which is to be produced as truly hd. Suppose that through enumeration of eigenvalues the sequence $\left\{\lambda_{m}\right\} \geq 0(m=1, \ldots, n)$ is made increasing. Then, matrix $\mathbf{G}_{n}$ given by (2.21) will be truly hyperdominant if $\lambda_{1}$ is sufficiently great.

Proof. Since by assumption the conditions of Theorem 3.3 (Case 1) are satisfied, then $\mathbf{G}_{n}$ produced by using (2.21) has hd sign pattern. As it is $\varepsilon_{k}=\left(\lambda_{k}^{*}-\lambda_{k+1}\right) a_{k}(k=1, \ldots, n-1)$, then from (2.17)-(2.18), (2.21) it follows that hd margin $p_{m}$ of the $m$ th row (or column) from $\mathbf{G}_{n}(m=1, \ldots, n)$ can be in general represented as

$$
\begin{gathered}
p_{m}=\alpha_{1}^{(m)} \lambda_{1}+\alpha_{2}^{(m)} \lambda_{2}+\cdots+\alpha_{m}^{(m)} \lambda_{m}+\alpha_{m+1}^{(m)} \lambda_{m+1} \quad(m=1, \ldots, n-1), \\
p_{n}=\alpha_{1}^{(n)} \lambda_{1}+\alpha_{2}^{(n)} \lambda_{2}+\cdots+\alpha_{n-1}^{(n)} \lambda_{n-1}+\alpha_{n}^{(n)} \lambda_{n},
\end{gathered}
$$

where " $\alpha$ " coefficients are defined as follows:

$$
\begin{gathered}
\alpha_{1}^{(1)}:=a_{1}\left(a_{1}+a_{2} c_{1}+a_{3} c_{2} c_{1}+\cdots+a_{n-1} c_{n-2} \cdots c_{2} c_{1}+c_{n-1} c_{n-2} \cdots c_{2} c_{1}\right), \quad m=1, \\
\alpha_{2}^{(1)}:=b_{1}\left(b_{1}+a_{2} d_{1}+a_{3} c_{2} d_{1}+\cdots+a_{n-1} c_{n-2} \cdots c_{2} d_{1}+c_{n-1} c_{n-2} \cdots c_{2} d_{1}\right), \\
\alpha_{1}^{(m)}:=\left(a_{m} c_{m-1} \cdots c_{2} c_{1}\right)\left(a_{1}+a_{2} c_{1}+a_{3} c_{2} c_{1}+\cdots+a_{m} c_{m-1} \cdots c_{2} c_{1}\right. \\
\left.+\cdots+a_{n-1} c_{n-2} \cdots c_{2} c_{1}+c_{n-1} c_{n-2} \cdots c_{2} c_{1}\right),
\end{gathered}
$$




$$
\begin{aligned}
& \alpha_{2}^{(m)}:=\left(a_{m} c_{m-1} \cdots c_{2} d_{1}\right)\left(b_{1}+a_{2} d_{1}+a_{3} c_{2} c_{1}+\cdots+a_{m} c_{m-1} \cdots c_{2} d_{1}+\cdots+a_{n-1} c_{n-2} \cdots c_{2} d_{1}\right. \\
& \left.+c_{n-1} c_{n-2} \cdots c_{2} d_{1}\right), \quad p=3, \ldots, m, \\
& \alpha_{p}^{(m)}:=\left(a_{m} c_{m-1} \cdots c_{p} d_{p-1}\right)\left(b_{p-1}+a_{p} d_{p-1}+a_{p+1} c_{p} d_{p-1}+\cdots+a_{n-1} c_{n-2} \cdots c_{p} d_{p-1}\right. \\
& \left.+c_{n-1} c_{n-2} \cdots c_{p} d_{p-1}\right), \quad m=2, \ldots, n-1, p=3, \ldots, m \\
& \alpha_{m}^{(m)}:=\left(a_{m} d_{m-1}\right)\left(b_{m-1}+a_{m} d_{m-1}+a_{m+1} c_{m} d_{m-1}+\cdots+a_{n-1} c_{n-2} \cdots c_{m} d_{m-1}+c_{n-1} c_{n-2} \cdots c_{m} d_{m-1}\right) \text { ， } \\
& \alpha_{m+1}^{(m)}:=b_{m}\left(b_{m}+a_{m+1} d_{m}+a_{m+2} c_{m+1} d_{m}+\cdots+a_{n-1} c_{n-2} \cdots c_{m+1} d_{m}+c_{n-1} c_{n-2} \cdots c_{m+1} d_{m}\right), \\
& m=2, \ldots, n-2 \text {, } \\
& \alpha_{n}^{(n-1)}:=b_{n-1}\left(b_{n-1}+d_{n-1}\right) \\
& \alpha_{1}^{(n)}:=\left(c_{n-1} c_{n-2} \cdots c_{2} c_{1}\right)\left(a_{1}+a_{2} c_{1}+a_{3} c_{2} c_{1}+\cdots+a_{n-1} c_{n-2} \cdots c_{2} c_{1}+c_{n-1} c_{n-2} \cdots c_{2} c_{1}\right), \\
& \alpha_{2}^{(n)}:=\left(c_{n-1} c_{n-2} \cdots c_{2} d_{1}\right)\left(b_{1}+a_{2} d_{1}+a_{3} c_{2} d_{1}+\cdots+a_{n-1} c_{n-2} \cdots c_{2} d_{1}+c_{n-1} c_{n-2} \cdots c_{2} d_{1}\right) \\
& \alpha_{p}^{(n)}:=\left(c_{n-1} c_{n-2} \cdots c_{p} d_{p-1}\right)\left(b_{p-1}+a_{p} d_{p-1}+a_{p+1} c_{p} d_{p-1}+\cdots+a_{n-1} c_{n-2} \cdots c_{p} d_{p-1}\right. \\
& \left.+c_{n-1} c_{n-2} \cdots c_{p} d_{p-1}\right), \quad p=3, \ldots, n-1 \text {. } \\
& \alpha_{n}^{(n)}:=d_{n-1}\left(b_{n-1}+d_{n-1}\right) .
\end{aligned}
$$

According to Case 1 of Theorem 3.3, both $a_{k}$ and $c_{k}$ are nonnegative when $\theta_{k} \in[0, \pi / 2](k=$ $1, \ldots, n-1)$. Then, from $(4.2)$ we see that $\alpha_{1}^{(m)} \geq 0(m=1, \ldots, n)$, whereas other " $\alpha$ "s may be nonpositive. Since " $\alpha$ "s depend only on selection of " $\theta$ "s, then by presuming $\lambda_{1}=\lambda_{2}=$ $\cdots=\lambda_{n}=\lambda \neq 0$, we obtain from $(2.21) \mathbf{G}_{n}=\lambda \mathbf{I}_{n}$ and $p_{m}=\lambda(m=1, \ldots, n)$ and from (4.1) we conclude that in general it holds:

$$
\sum_{p=1}^{m+1} \alpha_{p}^{(m)} \equiv 1 \quad(m=1, \ldots, n-1), \quad \sum_{q=1}^{n} \alpha_{q}^{(n)} \equiv 1 .
$$

Although $\mathbf{G}_{n}$ is produced with hd sign pattern, it will not be truly hd unless each of its row (column) hd margins is nonnegative, that is, $\mathbf{p}=\left[\begin{array}{ll}p_{1} & p_{2} \cdots p_{n}\end{array}\right]^{\mathrm{T}} \geq \mathbf{0}_{n, 1}$. The column vector $\mathbf{p}$ with entries (4.1) can be written as

$\mathbf{p}=\left[\begin{array}{cccccc}\alpha_{1}^{(1)} & \alpha_{2}^{(1)} & 0 & 0 & \cdots & 0 \\ \alpha_{1}^{(2)} & \alpha_{2}^{(2)} & \alpha_{3}^{(2)} & 0 & \cdots & 0 \\ \cdots & \cdots & \cdots & \cdots & \cdots & \cdots \\ \alpha_{1}^{(n-1)} & \alpha_{2}^{(n-1)} & \alpha_{3}^{(n-1)} & \cdots & \cdots & \alpha_{n}^{(n-1)} \\ \alpha_{1}^{(n)} & \alpha_{2}^{(n)} & \alpha_{3}^{(n)} & \cdots & \cdots & \alpha_{n}^{(n)}\end{array}\right]\left[\begin{array}{ccccccccc}1 & 0 & 0 & \cdots & \cdots & \cdots & 0 & 0 & 0 \\ -1 & 1 & 0 & \cdots & \cdots & \cdots & 0 & 0 & 0 \\ 0 & -1 & 1 & \cdots & \cdots & \cdots & 0 & 0 & 0 \\ \cdots & \cdots & \cdots & \cdots & \cdots & \cdots & \cdots & \cdots & \cdots \\ 0 & 0 & 0 & 0 & \cdots & \cdots & -1 & 1 & 0 \\ 0 & 0 & 0 & 0 & 0 & \cdots & 0 & -1 & 1\end{array}\right]^{-1}\left[\begin{array}{c}\lambda_{1} \\ \lambda_{2}-\lambda_{1} \\ \lambda_{3}-\lambda_{2} \\ \cdots \\ \lambda_{n-1}-\lambda_{n-2} \\ \lambda_{n}-\lambda_{n-1}\end{array}\right]$

$$
=\left[\begin{array}{cccccc}
1 & \alpha_{2}^{(1)} & 0 & 0 & \cdots & 0 \\
1 & \sum_{r=2}^{3} \alpha_{r}^{(2)} & \alpha_{3}^{(2)} & 0 & \cdots & 0 \\
\cdots & \cdots & \cdots & \cdots & \cdots & \cdots \\
1 & \sum_{s=2}^{n} \alpha_{s}^{(n-1)} & \sum_{u=3}^{n} \alpha_{u}^{(n-1)} & \cdots & \cdots & \alpha_{n}^{(n-1)} \\
1 & \sum_{t=2}^{n} \alpha_{t}^{(n)} & \sum_{w=3}^{n} \alpha_{w}^{(n)} & \cdots & \cdots & \alpha_{n}^{(n)}
\end{array}\right]\left[\begin{array}{c}
\lambda_{1} \\
\lambda_{2}-\lambda_{1} \\
\lambda_{3}-\lambda_{2} \\
\cdots \\
\lambda_{n-1}-\lambda_{n-2} \\
\lambda_{n}-\lambda_{n-1}
\end{array}\right] .
$$


From (4.4) we finally obtain

$$
\begin{aligned}
& p_{1}=\lambda_{1}+\left(\lambda_{2}-\lambda_{1}\right) \alpha_{2}^{(1)}, \quad p_{2}=\lambda_{1}+\left(\lambda_{2}-\lambda_{1}\right) \sum_{r=2}^{3} \alpha_{r}^{(2)}+\left(\lambda_{3}-\lambda_{2}\right) \alpha_{3}^{(2)}, \ldots, \\
& p_{n}=\lambda_{1}+\left(\lambda_{2}-\lambda_{1}\right) \sum_{t=2}^{n} \alpha_{t}^{(n)}+\left(\lambda_{3}-\lambda_{2}\right) \sum_{w=3}^{n} \alpha_{w}^{(n)}+\cdots+\left(\lambda_{n}-\lambda_{n-1}\right) \alpha_{n}^{(n)} .
\end{aligned}
$$

Since " $\alpha$ "s and " $\Sigma$ "s in (4.5) are not certainly nonnegative and since the sequence $\left\{\lambda_{m}\right\} \quad(m=$ $1, \ldots, n)$ is increasing, then firstly by arbitrary selection of differences $\lambda_{2}-\lambda_{1} \geq 0, \lambda_{3}-\lambda_{2} \geq$ $0, \ldots, \lambda_{n-1}-\lambda_{n-2} \geq 0$ and $\lambda_{n}-\lambda_{n-1} \geq 0$ and thereafter a sufficiently great $\lambda_{1} \geq 0$, all hd margins $p_{m}(m=1, \ldots, n)$ can be made nonnegative, that is, the matrix $\mathbf{G}_{n}$ can be always produced as truly hyperdominant. This completes the proof of this theorem.

Presentation of explicit solution to the IEP of truly hd matrices with assigned nonnegative spectrum is now in order. It has been proved in [16] that this IEP always has at least one solution and that infinitely many others can be produced thereof by using Givens rotations. Solution of that IEP is important in electrical network synthesis of drivingpoint immittance functions and matrices of both passive and active, common-ground, transformerless, two-element-kind RLC networks and in generation of various classes of canonic and noncanonic equivalent realizations $[19,22]$. In [16] we have proved the existence of solution to the IEP of hd matrices with assigned nonnegative spectrum, but here we shall present the explicit construction of solution matrix entries by using other arguments. This represents the explicit solution of the problem opened in [17].

Theorem 4.2. For any set of real nonnegative numbers $\left\{\lambda_{1}, \lambda_{2}, \ldots, \lambda_{n}\right\}$ there always exists at least one (and infinitely many) $n \times n$ real symmetric hyperdominant matrices having these numbers as eigenvalues. In other words, IEP of symmetric $h d$ matrices with assigned nonnegative spectrum always has at least one solution.

Proof. We will take the same assumptions as in Theorem 4.1, except for $\theta_{k} \in(0, \pi / 2)(k=$ $1, \ldots, n-1)$. Through enumeration of eigenvalues, the nonnegative sequence $\left\{\lambda_{m}\right\}$ ( $m=$ $1, \ldots, n)$ is made increasing. Then, according to Theorem 3.3 (Case 1 ), the symmetric matrix $\mathbf{G}_{n}=\mathbf{U G}_{1} \mathbf{U}^{\mathrm{T}}\left[\mathbf{G}_{1}=\operatorname{diag}\left(\lambda_{1}, \lambda_{2}, \ldots, \lambda_{n}\right)\right]$ with spectrum $\left\{\lambda_{1}, \lambda_{2}, \ldots, \lambda_{n}\right\}$ and the entries determined by (2.21), is produced with hd sign pattern, no matter what selection of $\theta_{k} \mathrm{~s}(k=$ $1, \ldots, n-1)$ has been made. Observe that in Case $1 a_{k}=\cos \theta_{k}$ and $c_{k}=\sin \theta_{k}$. To make $\mathbf{G}_{n}=\mathbf{U G}_{1} \mathbf{U}^{\mathrm{T}}$ truly hd, we will prove the existence of such " $\theta$ "s that make all " $\alpha$ "s (and hence all " $p$ "s) in (4.1) nonnegative. Let we introduce the following positive sequence $\left\{M_{m}\right\}(m=1, \ldots, n)$

$$
\begin{aligned}
M_{1} & :=a_{1}+a_{2} c_{1}+a_{3} c_{2} c_{1}+\cdots+a_{n-1} c_{n-2} \cdots c_{2} c_{1}+c_{n-1} c_{n-2} \cdots c_{2} c_{1} \\
M_{2} & :=a_{2}+a_{3} c_{2}+a_{4} c_{3} c_{3}+\cdots+a_{n-1} c_{n-2} \cdots c_{3} c_{2}+c_{n-1} c_{n-2} \cdots c_{3} c_{2} \\
M_{3} & :=a_{3}+a_{4} c_{3}+a_{5} c_{4} c_{3}+\cdots+a_{n-1} c_{n-2} \cdots c_{4} c_{3}+c_{n-1} c_{n-2} \cdots c_{4} c_{3} \\
M_{n-2} & :=a_{n-2}+a_{n-1} c_{n-2}+c_{n-1} c_{n-2} \\
M_{n-1} & :=a_{n-1}+c_{n-1} \\
M_{n} & :=1 .
\end{aligned}
$$


Then, by using (4.2) we obtain a consistent set of inequalities that ensure nonnegativity of all " $\alpha$ "s in (4.1)

$$
\begin{gathered}
\alpha_{1}^{(1)}=a_{1} M_{1} \geq 0, \quad \alpha_{2}^{(1)}=1-a_{1} M_{1}=c_{1}\left(c_{1}-a_{1} M_{2}\right) \geq 0, \\
\alpha_{1}^{(k)} \geq 0, \quad \alpha_{p}^{(k)} \geq 0 \Longleftrightarrow-c_{p-1}+a_{p-1} M_{p} \geq 0 \quad(p=2, \ldots, k), \\
\alpha_{k+1}^{(k)} \geq 0 \Longleftrightarrow c_{k}-a_{k} M_{k+1} \geq 0 \quad(k=2, \ldots, n-1), \\
\alpha_{1}^{(n)} \geq 0, \quad \alpha_{q}^{(n)} \geq 0 \Longleftrightarrow-c_{q-1}+a_{q-1} M_{q} \geq 0 \quad(q=2, \ldots, n-1), \\
\alpha_{n}^{(n)} \geq 0 \Longleftrightarrow-c_{n-1}+a_{n-1} \geq 0, \\
\alpha_{n}^{(n-1)}=b_{n-1}^{2}+b_{n-1} d_{n-1}=c_{n-1}^{2}-a_{n-1} c_{n-1}=c_{n-1}\left(c_{n-1}-a_{n-1}\right) \geq 0 \Longleftrightarrow c_{n-1}-a_{n-1} \geq 0 .
\end{gathered}
$$

For $p=2$ from (4.8) we obtain $M_{2} \geq c_{1} / a_{1}$ and from (4.7) $M_{2} \leq c_{1} / a_{1}$. Then, $M_{2}=$ $c_{1} / a_{1}, \alpha_{2}^{(1)}=0, \alpha_{1}^{(1)}=1-\alpha_{2}^{(1)}=1, M_{1}=1 / a_{1}$ and $p_{1}=\lambda_{1}$. From (4.11)-(4.12) it follows that $a_{n-1}=c_{n-1}\left(\leftrightarrow \theta_{n-1}=\pi / 4\right)$ and $\alpha_{n}^{(n-1)}=\alpha_{n}^{(n)}=0$ [inequalty (4.12) is the same as (4.9) if $k=n-1\left(M_{n}=1\right)$ ]. For $k=2, \ldots,(n-2)$, we obtain from (4.9) $M_{k+1} \leq c_{k} / a_{k}$ and for $q=3, \ldots,(n-1)$, we obtain from (4.10) $M_{q} \geq c_{q-1} / a_{q-1}$. To summarize, we have proved that: (a) $M_{r}=c_{r-1} / a_{r-1}$, for $r=2, \ldots,(n-1)$ and (b) $\left\{\alpha_{1}^{(k)}=1, \alpha_{s}^{(k)}=0[s=2, \ldots,(k+1)]\right.$ and $\left.p_{k}=\lambda_{1}\right\}$, for $k=1, \ldots,(n-1)$. And finally, from (4.10) we obtain $\alpha_{q}^{(n)}=0$ for $q=2, \ldots, n, \alpha_{1}^{(n)}=1$ and $p_{n}=\lambda_{1}$. Since the matrix $\mathrm{G}_{n}$ has hd sign pattern and each of its row (column) hd margins is equal to $\lambda_{1} \geq 0$, then $\mathbf{G}_{n}$ is truly hd matrix. This completes the proof of the theorem.

Remark 4.3. It relates to calculation of entries of $\mathbf{G}_{n}$. In Theorem 4.2 it is proved that $M_{1}=$ $1 / a_{1}$ and $M_{k}=c_{k-1} / a_{k-1}(k=2, \ldots, n-1)$. It is assumed $M_{n}=1$. Since $\theta_{n-1}=\pi / 4$, then $M_{n-1}=a_{n-1}+c_{n-1}=\cos \theta_{n-1}+\sin \theta_{n-1}=\sqrt{2}$. For $w=1, \ldots, n-1$ it follows from (4.6)

$$
\begin{aligned}
M_{w} & =a_{w}+c_{w} M_{w+1}=a_{w}+c_{w} \frac{c_{w}}{a_{w}}=\frac{1}{a_{w}}=\sqrt{\frac{a_{w}^{2}+c_{w}^{2}}{a_{w}^{2}}}=\sqrt{1+\left(\frac{c_{w}}{a_{w}}\right)^{2}} \\
& =\sqrt{1+M_{w+1}^{2}}=\sqrt{2+M_{w+2}^{2}}=\cdots=\sqrt{n-w-1+M_{n-1}^{2}}=\sqrt{n-w+1}, \\
a_{w} & =\cos \theta_{w}=\frac{1}{\sqrt{n-w+1}}, \quad c_{w}=\sin \theta_{w}=\sqrt{\frac{n-w}{n-w+1}} .
\end{aligned}
$$

By using (2.17)-(2.18), (2.21), (4.13) we can easily calculate all entries of the (initial) hd matrix $\mathrm{G}_{n}$. Other hd matrices having the same spectrum can be produced thereof by application of Givens rotations, one at a time.

\section{Application of the obtained results in electrical network synthesis}

It is well known that synthesis methods of passive, common-ground, transformerless, twoelement-kind $R L C$ networks yield topological configurations which are severely restricted by the method chosen [19]. By using of the results above, a new class of non-canonic, drivingpoint immittance realizations of passive, common-ground, transformerless, two-elementkind RLC networks with minimum number of both nodes and elements of one kind can 
be generated with possibility of reduction in number of elements of other kind. The network synthesis is always performed by using normalization of both the complex frequency $s$ and the impedance $Z(s)$. If $\Omega$ is a selected normalization frequency, then the normalized frequency is $s_{n}=s / \Omega$. Similarly, if $R_{0}$ is a selected normalization resistance, then the normalized impedance is $Z_{n}(s)=Z(s) / R_{0}$. Thereby we achieve [20]: (a) lesser dispersion of coefficients in normalized functions and (b) dimensionless manipulation of quantities. The normalized resistance of resistor $R$ is $R_{n}:=R / R_{0}$. The normalized impedance of an inductor $L$ is $Z_{L n}(s)=L s / R_{0}=\left(L \Omega / R_{0}\right) s_{n}=L_{n} s_{n}\left(L_{n}:=L \Omega / R_{0}\right.$-normalized inductance). The normalized impedance of a capacitor $C$ is $Z_{C n}(s)=1 /\left(C R_{0} s\right)=1 /\left[\left(R_{0} C \Omega\right) s_{n}\right]=1 /\left(C_{n} s_{n}\right)\left(C_{n}:=R_{0} C \Omega\right.$ normalized capacitance). To physically realize a network after synthesis, a denormalization process must be performed. The actual parameter values of $R L C$ elements are calculated as follows: $R=R_{n} R_{0}, L=L_{n} R_{0} / \Omega, C=C_{n} /\left(R_{0} \Omega\right)$. From now on it will be assumed that normalized synthesis is being carried out, but the lower index " $n$ " we be dropped from component labels for brevity.

It is well known that if a real rational function in $s$ can be realized as $R L$ drivingpoint impedance $Z_{R L}(s)$, then it can be also realized as $R C$ driving-point admittance $Y_{R C}(s)$ [20]. And similarly, if it can be realized as $R L$ driving-point admittance $Y_{R L}(s)$, then it can also be realized as $R C$ driving-point impedance $Z_{R C}(s)$. The $L C: R C$ transformation turns the synthesis of $L C$ driving-point impedance $Z_{L C}^{\square}(s)$ to synthesis of $R C$ driving-point impedance $Z_{R C}(s)=Z_{L C}^{\square}(\sqrt{s}) / \sqrt{s}$ [20]. It also turns the synthesis of $L C$ driving-point admittance $Y_{L C}^{\square}(s)$ to synthesis of $R C$ driving-point admittance $Y_{R C}(s)=\sqrt{s} \cdot Y_{L C}^{\square}(\sqrt{s})$. These $R C$ driving-point imittances are at first realized by prototype $R C$ networks and thereof are produced the desired $L C$ networks in the following way: capacitors in $R C$ and $L C$ networks remain the same, but the resistor from $R C$ network turns to inductor in $L C$ network with the same parameter value. Also, $L R: R C$ transformation turns the synthesis of $R L$ drivingpoint impedance $Z_{R L}^{\square}(s)$ to synthesis of $R C$ driving-point impedance $Z_{R C}(s)=Z_{R L}^{\square}(s) / s$. It also turns synthesis of $R L$ driving-point admittance $Y_{R L}^{\square}(s)$ to synthesis of $R C$ driving-point admittance $Y_{R C}(s)=s Y_{R L}^{\square}(s)$. These $R C$ imittances are realized by prototype $R C$ networks and the desired $R L$ networks are produced thereof in the following way: the resistor from $R C$ network turns to inductor in $L R$ network with the same parameter value, and the capacitor from $R C$ network turns to resistor in $L R$ network with reciprocal parameter value. Bearing all the aforementioned on mind, we can obviously restrict our consideration only to synthesis of driving-point impedance functions $Z_{R C}(s)$ of $R C$ networks, which satisfy the following well known analytic necessary and sufficient conditions [20]: (a) $Z_{R C}(s)$ is real rational function in $s$, (b) It has only simple poles on negative real axis, or at the origin. At infinity it cannot have pole and (c) Residues of these poles are real and positive and $A_{\infty}:=\lim _{s \rightarrow \infty} Z_{R C}(s) \geq 0$. In general, the first canonic Foster's expansion (form) of $Z_{R C}(s)$ [20] reads

$$
Z_{R C}(s)=A_{\infty}+\sum_{m=0}^{n} \frac{A_{m}}{s+s_{m}} \quad\left[A_{\infty}>0 ; s_{0}=0, A_{0}>0 ; s_{p}, A_{p}>0(p=1, \ldots, n)\right]
$$

where $A_{m}$ is residue of the pole $s_{m}(m=0,1, \ldots, n)$. The network which realizes drivingpoint impedance $Z_{R C}(s)$ (5.1) with minimum number of nodes $(=n+1)$, minimum number of resistors $(=n+1)$ and minimum number of capacitors $(=n+1)$ is depicted in Figure 1. Observe that neither the resistors, nor the capacitors share common-node and hence the overall network realization is said to be non common-grounded.

Now, we will present our synthesis procedure. If for a given driving-point impedance $Z_{R C}(s)$ we found that $A_{0}>0$ and/or $A_{\infty}>0$, then in the preamble of the realization 


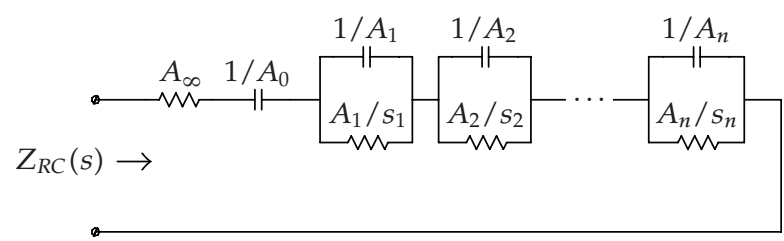

Figure 1: The first canonic Foster's realization of $Z_{R C}(s)$. Denoted are the normalized "values" of $R C$ parameters.

procedure $A_{\infty}$ and/or $A_{0} / s$ should be at first extracted from (5.1) and realized by a series connection of resistor $R_{\infty}=A_{\infty}$ and capacitor $C_{0}=1 / A_{0}$, thereby leaving for realization the driving-point impedance $\bar{Z}_{R C}(s)=Z_{R C}(s)-A_{\infty}-A_{0} / s$ with solely $n$ poles lying on the negative real axis. In the sequel we will assume that $Z_{R C}(s)$ has only $n$ such poles.

Let $\mathbf{C}=\operatorname{diag}\left(C_{1}, C_{2}, \ldots, C_{n}\right)$ and $\mathbf{G}=\operatorname{diag}\left(G_{1}, G_{2}, \ldots, G_{n}\right)$ be diagonal $n \times n$ matrices with strictly positive diagonal entries corresponding to the normalized capacitances and conductances, respectively. If we arbitrarily choose a nonsingular $n \times n$ matrix $\mathbf{T}$, then the reciprocal passive networks which realize $\mathbf{C} s+\mathbf{G}$ and $\mathbf{Y}(s)=\mathbf{T}(\mathbf{C} s+\mathbf{G}) \mathbf{T}^{\mathrm{T}}$ will have the same natural frequencies. By arbitrary selection of $n \times n$ nonsingular diagonal matrices $\delta=\operatorname{diag}\left(\delta_{1}, \delta_{2}, \ldots, \delta_{n}\right)$, a broad class of nonsingular $n \times n$ matrices $\mathbf{T}$ can be generated with assumption $\mathbf{T}=\mathbf{V} \boldsymbol{\delta} \mathbf{U}$, where $\mathbf{U}$ and $\mathbf{V}$ are $n \times n$ orthogonal matrices. Since $\mathbf{Y}(s)=$ $\mathrm{T}(\mathrm{C} s+\mathrm{G}) \mathrm{T}^{\mathrm{T}}=\mathbf{V}\left[\boldsymbol{\delta}\left(\mathbf{U C \mathbf { U } ^ { \mathrm { T } }}\right) \boldsymbol{\delta} s+\boldsymbol{\delta}\left(\mathbf{U G U}^{\mathrm{T}}\right) \boldsymbol{\delta}\right] \mathbf{V}^{\mathrm{T}}$, then

$$
\mathbf{Z}(s)=\left[z_{m r}(s)\right]_{n \times n}=\mathbf{Y}^{-1}(s)=\left[\mathbf{T}(\mathbf{C} s+\mathbf{G}) \mathbf{T}^{\mathrm{T}}\right]^{-1}=\left(\mathbf{V} \boldsymbol{\delta}^{-1} \mathbf{U} \mathbf{C}^{1 / 2}\right)\left(s \mathbf{I}_{n}+\mathbf{G C}^{-1}\right)^{-1}\left(\mathbf{V} \boldsymbol{\delta}^{-1} \mathbf{U} \mathbf{C}^{1 / 2}\right)^{\mathrm{T}}
$$

Various network topologies can be produced by different choices of $\mathbf{U}$ and $\mathbf{V}$. But, only by selecting $\mathbf{C}=C \mathbf{I}_{n}(C>0)$ and $\mathbf{V}=\mathbf{I}_{n}$, the networks with minimum number of commonground capacitors are produced; and only by selecting $\mathbf{G}=G \mathbf{I}_{n}(G>0)$ and $\mathbf{V}=\mathbf{I}_{n}$, the networks with minimum number of common-ground resitors are produced. Let us select $\mathbf{C}=C \mathbf{I}_{n}(C>0)$ and $\mathbf{V}=\mathbf{I}_{n}$, and let us assume in (5.1): $A_{0}=A_{\infty}=0$ and $s_{n}>s_{n-1} \cdots>s_{1}>0$. Since $\mathbf{U}=\left[u_{m r}\right](m, r=1, \ldots, n)$, then from (5.2) it follows that

$$
\mathbf{Y}(s)=C \boldsymbol{\delta}^{2} s+\boldsymbol{\delta}\left(\mathbf{U G U}^{\mathrm{T}}\right) \boldsymbol{\delta}, \quad z_{m r}(s)=\frac{1}{C \delta_{m} \delta_{r}} \sum_{p=1}^{n} \frac{u_{m p} u_{r p}}{s+G_{p} / C} \quad(m, r=1, \ldots, n) .
$$

The matrices which are effectively realized by common-ground network with $n+1$ nodes $[(n+1)$ th node is the common-ground $]$ are $C \boldsymbol{\delta}^{2} s$ and $\delta\left(\mathbf{U G U ^ { T }}\right) \boldsymbol{\delta}$, provided that both are truly hd. According to (5.1) and (5.3) it holds $G_{p}=C s_{p}(p=1, \ldots, n)$ and $G_{n}>G_{n-1} \cdots>G_{1}>0$. By using Theorem 3.3 [Case $1, \mathbf{P}_{k}=\mathbf{A}_{k}$ and $\theta_{k} \in(0, \pi / 2)(k=1, \ldots, n-1)$ ] we infer that UGU ${ }^{\mathrm{T}}$ (2.21) is produced with hd sign pattern and no zero entries and with strictly positive inverse. Matrix $\mathbf{U}(2.26)$ is lower Hessenberg with nonnegative entries, except for negative " $b$ "s. The same conclusions relating to $\mathbf{U G U}^{\mathrm{T}}$ and $\mathbf{U}$ also hold if we apply Case 1 of Theorem 3.3 with $\mathbf{P}_{k}=\mathbf{B}_{k}$ and $\theta_{k} \in(0, \pi / 2)(k=1, \ldots, n-1)$, except for " $d$ "s in (2.26) are then negative and " $b$ "s are positive. To realize $Z_{R C}(s)$ we must select in (5.3) either $m=r=n-1$ or $m=r=n$, thus obtaining either $Z_{R C}(s)=z_{n-1, n-1}(s)$, or $Z_{R C}(s)=z_{n n}(s)$. By assuming 
$m=r=n\left[\leftrightarrow Z_{R C}(s)=z_{n n}(s)\right], \mathbf{P}_{k}=\mathbf{A}_{k}$ and $\theta_{k} \in(0, \pi / 2)(k=1, \ldots, n-1)$, it follows from (2.26), (5.1), (5.3)

$$
C \delta_{n}^{2}=\frac{1}{\sum_{p=1}^{n} A_{p}}, \quad a_{i-1}=d_{i-1}=\left(\frac{A_{i}}{\sum_{q=1}^{i} A_{q}}\right)^{1 / 2}, \quad c_{i-1}=\left(\frac{\sum_{r=1}^{i-1} A_{r}}{\sum_{q=1}^{i} A_{q}}\right)^{1 / 2}, \quad(i=2, \ldots, n) .
$$

To prove the existence of a physical realization of both $C \boldsymbol{\delta}^{2} s$ and $\boldsymbol{\delta}\left(\mathbf{U G U}^{\mathrm{T}}\right) \boldsymbol{\delta}$ we still have to determine the positive column vector $\operatorname{col}(\boldsymbol{\delta})=\left[\begin{array}{ll}\delta_{1} & \delta_{2} \cdots \delta_{n}\end{array}\right]^{\mathrm{T}}$ which, according to Theorem 3.5, makes $\boldsymbol{\delta}\left(\mathbf{U G U}^{\mathrm{T}}\right) \boldsymbol{\delta}$ truly hd with possibly zero hd margins of at most $n-1$ rows.

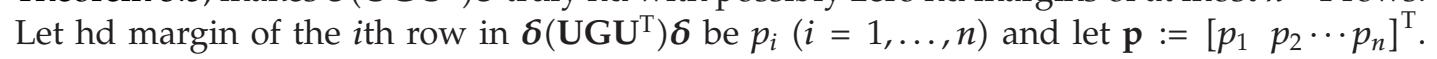
If $\mathbf{u}:=\left[\begin{array}{lll}1 & 1 & \cdots\end{array}\right]^{\mathrm{T}}(n$ unities $)$, then $\mathbf{p}=\boldsymbol{\delta}\left(\mathbf{U G U}^{\mathrm{T}}\right) \boldsymbol{\delta} \mathbf{u}$. Let we introduce a column vector $\mathbf{p}^{*}:=\left[\begin{array}{lll}p_{1}^{*} & p_{2}^{*} \cdots p_{n}^{*}\end{array}\right]^{\mathrm{T}}\left(\left\|\mathbf{p}^{*}\right\|_{\mathrm{E}}>0\right)$ of arbitrarily assumed real nonegative numbers. Since $\left(\mathbf{U G U}^{\mathrm{T}}\right)^{-1}$ is strictly positive, then it always can be find a diagonal matrix $\boldsymbol{\delta}=\operatorname{diag}\left(\delta_{1}, \delta_{2}, \ldots, \delta_{n}\right)$ with positive diagonal entries, such that $\mathbf{p}^{*}=\left(\mathbf{U G U}^{\mathrm{T}}\right) \boldsymbol{\delta} \mathbf{u}=\left(\mathbf{U G U}^{\mathrm{T}}\right) \operatorname{col}(\boldsymbol{\delta})$. Herefrom, we obtain $\operatorname{col}(\boldsymbol{\delta})=\left(\mathbf{U G U}^{\mathrm{T}}\right)^{-1} \mathbf{p}^{*}$, that is, that $\delta_{i}>0(i=1, \ldots, n)$. Since $\mathbf{p}=\boldsymbol{\delta}\left(\mathbf{U G U}^{\mathrm{T}}\right) \boldsymbol{\delta} \mathbf{u}=\boldsymbol{\delta} \mathbf{p}^{*}$, then it follows $p_{i}=\delta_{i} p_{i}^{*} \geq 0(i=1, \ldots, n)$, bearing on mind that at most $n-1$ " $p$ "s can be equal to zero. These " $p$ "s indices correspond to indices of those rows (or columns) in $\boldsymbol{\delta}\left(\mathbf{U G U}^{\mathrm{T}}\right) \boldsymbol{\delta}$ which have zero hd margins. Then, from the overall network vanish resistors connecting common-ground to nodes with the same indices as that of rows (columns) with zero hd margins [22]. For different selections of $\mathbf{p}^{*}$, different algorithms and different topologically and parametrically equivalent realizations emerge. For example, if we select $\operatorname{col}(\boldsymbol{\delta})=\mu\left[\begin{array}{lll}u_{11} & u_{21} \cdots u_{n 1}\end{array}\right]^{\mathrm{T}}(\mu>0)$, where it is according to $(2.26),(5.4)$

$$
u_{11}=\left[\frac{A_{2}}{A_{1}+A_{2}}\right]^{1 / 2}, \quad u_{p 1}=\left[\frac{A_{1} A_{p+1}}{\left(\sum_{q=1}^{n} A_{q}\right)\left(\sum_{r=1}^{p+1} A_{r}\right)}\right]^{1 / 2} \quad(p=2, \ldots, n-1), \quad u_{n 1}=\left[\frac{A_{1}}{\sum_{q=1}^{n} A_{q}}\right]^{1 / 2},
$$

then the column vector $\mathbf{p}$ of row (column) hd margins of matrix $\boldsymbol{\delta}\left(\mathbf{U G U}^{\mathrm{T}}\right) \boldsymbol{\delta}$ with hd sign pattern reads

$$
\mathbf{p}=\boldsymbol{\delta}\left(\mathbf{U G U}^{\mathrm{T}}\right) \boldsymbol{\delta} \mathbf{u}=\boldsymbol{\delta}\left(\mathbf{U G U}^{\mathrm{T}}\right) \operatorname{col}(\boldsymbol{\delta})=G_{1} \operatorname{col}\left(\boldsymbol{\delta}^{2}\right)>\mathbf{0}_{n, 1} .
$$

This means that $\boldsymbol{\delta}\left(\mathbf{U G U}^{\mathrm{T}}\right) \boldsymbol{\delta}$ is truly hd. We will now present two algorithms for realization of driving-point impedances $Z_{R C}(s)$ which rely on the results developed above.

Algorithm 1. Realization of $Z_{R C}(s)$ with minimum number of common-ground capacitors and non-reduced number of resistors and (5.4).

$\left(1^{0}\right)$ Commencing with $A_{i}(i=1, \ldots, n)$ calculate the entries of $\mathbf{U}$, by using (2.26) $1, \ldots, n)$.

$\left(2^{0}\right)$ Arbitrarily select some $\mu>0$ and then calculate $C=1 / \mu^{2} A_{1}$ and $G_{q}=C s_{q}(q=$

$\left(3^{0}\right)$ Calculate $\operatorname{col}(\boldsymbol{\delta})=\mu\left[\begin{array}{lll}u_{11} & u_{21} \cdots u_{n 1}\end{array}\right]^{\mathrm{T}}$, by using (5.5) and the entries of hd matrix $\boldsymbol{\delta}\left(\mathbf{U G U}^{\mathrm{T}}\right) \boldsymbol{\delta}$. Calculate $\mathrm{p}$, by using (5.6).

$\left(4^{0}\right)$ Realize $\boldsymbol{\delta}\left(\mathbf{U G U}^{\mathrm{T}}\right) \boldsymbol{\delta}$ by common-ground, transformerless, conductance network. This can be done easily, almost by visual inspection of $\boldsymbol{\delta}\left(\mathbf{U G U}^{\mathrm{T}}\right) \boldsymbol{\delta}$ [22]. Attach to the ports 


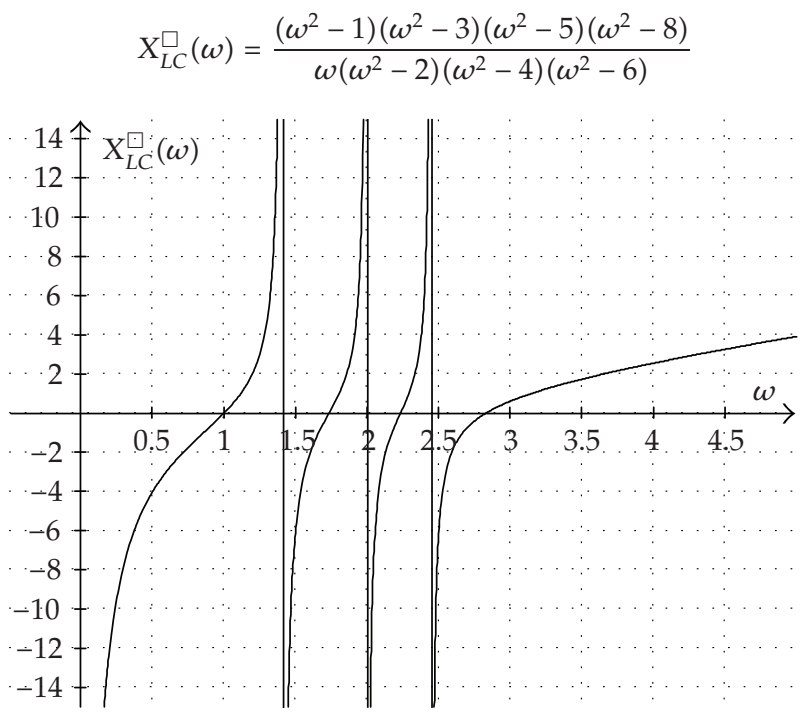

Figure 2: The reactance function of $L C$ network from the example.

of that network, enumerated by $1, p(p=2, \ldots, n-1)$ and $n$, the common-ground capacitors with normalized capacitances

$$
C_{1}=\frac{A_{2}}{A_{1}\left(A_{1}+A_{2}\right)}, \quad C_{p}=\frac{A_{p+1}}{\left(\sum_{q=1}^{n} A_{q}\right)\left(\sum_{r=1}^{p+1} A_{r}\right)} \quad(p=2, \ldots, n-1), \quad C_{n}=\frac{1}{\sum_{q=1}^{n} A_{q}}
$$

respectively. The $n$th port of the overall network realizes driving-point impedance $Z_{R C}(s)$, provided that all other ports are left open-circuited.

Algorithm 2. Realization of $Z_{R C}(s)$ with minimum number of common-ground capacitors and the reduced number of resistors

$\left(1^{0}\right)$ The same as step $\left(1^{0}\right)$ of Algorithm 1. Let $\mathbf{S}=\operatorname{diag}\left(s_{1}, s_{2}, \ldots, s_{n}\right)$. Recall that $s_{n}>$ $s_{n-1} \cdots>s_{1}>0$. calculate

$\left(2^{0}\right)$ Select $\varepsilon_{1}>0$ and $\varepsilon_{i}=0(i=2, \ldots, n-1)$. Thereafter, by using (2.21) and (5.4),

$$
C=\left[\varepsilon_{1}\left(s_{1}^{-1}-s_{2}^{-1}\right) c_{n-1} c_{n-2} \cdots c_{2} c_{1} a_{1}\right]^{2} \sum_{q=1}^{n} A_{q}, \quad G_{i}=C s_{i} \quad(i=1, \ldots, n) .
$$

Calculate $\operatorname{col}(\boldsymbol{\delta})=\varepsilon_{1} C^{-1}\left(\mathbf{U S}^{-1} \mathbf{U}^{\mathrm{T}}\right) \mathbf{e}_{1}$, where $\mathbf{e}_{1}=\left[\begin{array}{lll}1 & 0 & \cdots\end{array}\right]^{\mathrm{T}}$ is $n$-dimensional column vector.

$\left(3^{0}\right)$ Calculate the entries of $\boldsymbol{\delta}\left(\mathbf{U G U}^{\mathrm{T}}\right) \boldsymbol{\delta}$ and its hd margin $p_{1}=\delta_{1} \varepsilon_{1}$, where $\delta_{1}=$ $\varepsilon_{1} C^{-1} \mathbf{e}_{1}^{\mathrm{T}} \mathbf{T S}^{-1} \mathbf{T}^{\mathrm{T}} \mathbf{e}_{1}$. Set for other hd margins $p_{i}=0(i=2, \ldots, n)$.

$\left(4^{0}\right)$ Realize $\boldsymbol{\delta}\left(\mathbf{U G U}^{\mathrm{T}}\right) \boldsymbol{\delta}$ by common-ground, transformerless, conductance network and attach to its $i$ th port the common-ground capacitor with normalized capacitance $C \delta_{i}^{2}(i=$ $1, \ldots, n)$. The $n$th port of the overall network realizes driving-point impedance $Z_{R C}(s)$, provided that all other ports are left open-circuited. 


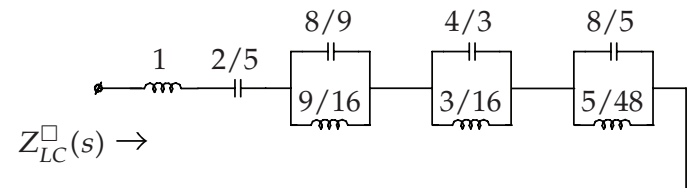

Figure 3: Driving-point impedance $Z_{L C}^{\square}(s)$ from example synthesized by using the first canonic Foster's form.

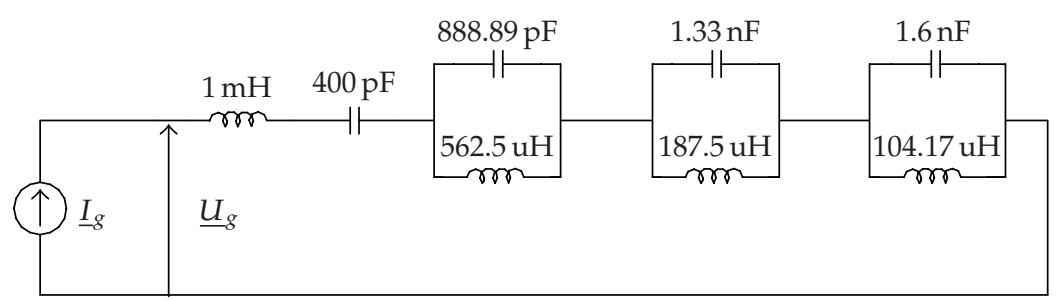

Figure 4: Denormalized realization of driving-point impedance $Z_{L C}^{\square}(s)$ from Figure 3.

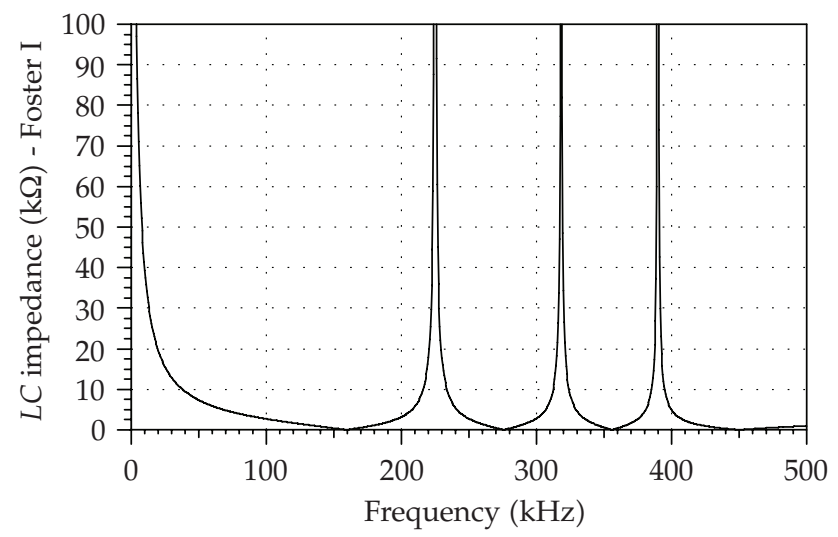

Figure 5: Driving-point impedance $\left|\underline{Z}_{L C}^{\square}\right|$ of $L C$ network from Figure 4.

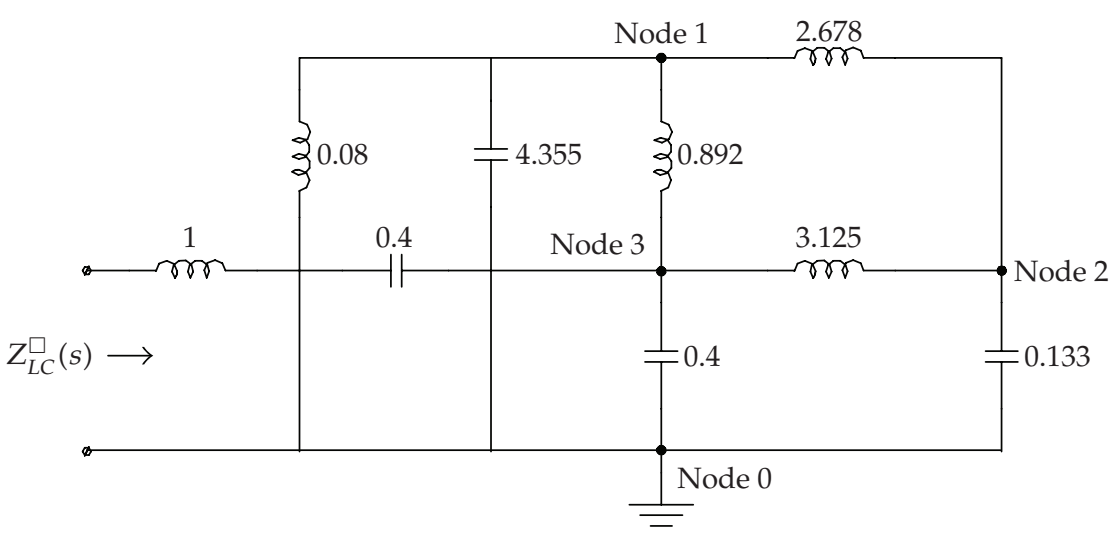

Figure 6: Driving-point impedance $Z_{L C}^{\square}(s)$ from the example synthesized by a noncanonic $L C$ network. 


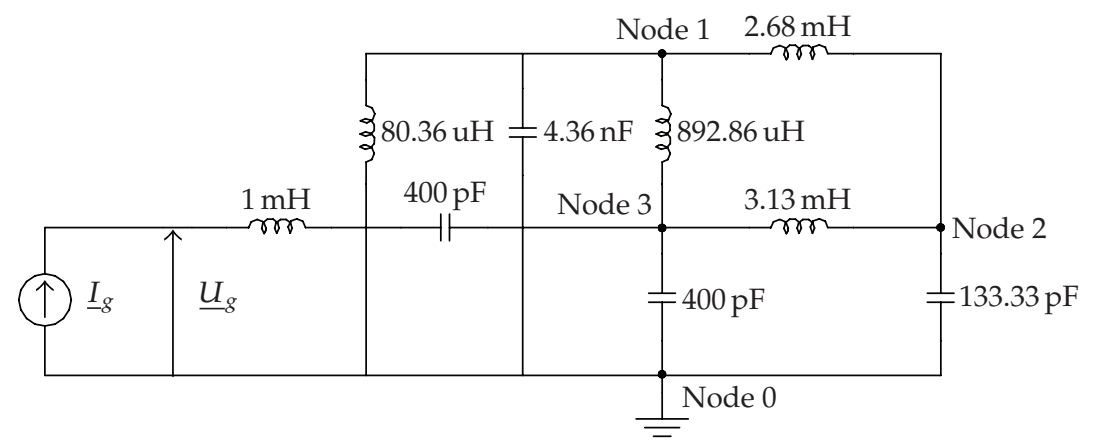

Figure 7: Denormalized realization of driving-point impedance $Z_{L C}^{\square}(s)$ from Figure 6 .

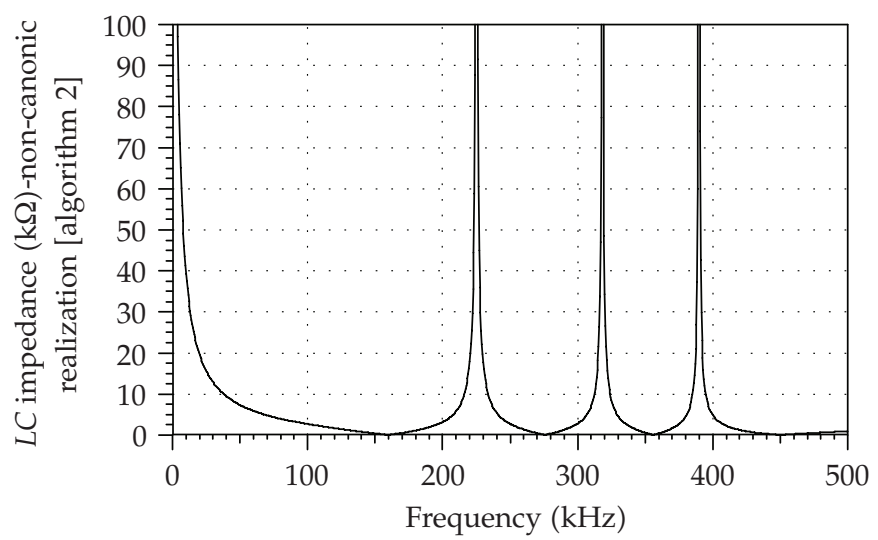

Figure 8: Driving-point impedance $\left|\underline{Z}_{L C}^{\square}\right|$ of $L C$ network from Figure 7.

\subsection{A numerical example}

Consider realization of the real rational function $Z_{L C}^{\square}(s)$ as driving-point impedance of common-ground transformerless $L C$ network with minimum number of capacitors and the reduced number of inductors

$$
Z_{L C}^{\square}(s)=\frac{\left(s^{2}+1\right)\left(s^{2}+3\right)\left(s^{2}+5\right)\left(s^{2}+8\right)}{s\left(s^{2}+2\right)\left(s^{2}+4\right)\left(s^{2}+6\right)} .
$$

This function satisfies the necessary and sufficient conditions for driving-point immittances of $L C$ networks: (a) it is an odd real rational function in $s ;(b)$ it has only simple poles located strictly on imaginary axis; and (c) residues of those poles are real and positive. Therefore, $Z_{L C}^{\square}(s)$ can be realized both in two Foster's and in two Cauer's canonic forms [20]. The partial fraction expansion of $Z_{L C}^{\square}(s)$ reads

$$
Z_{L C}^{\square}(s)=\frac{\left(s^{2}+1\right)\left(s^{2}+3\right)\left(s^{2}+5\right)\left(s^{2}+8\right)}{s\left(s^{2}+2\right)\left(s^{2}+4\right)\left(s^{2}+6\right)}=s+\frac{5}{2 s}+\frac{9 s / 8}{s^{2}+2}+\frac{3 s / 4}{s^{2}+4}+\frac{5 s / 8}{s^{2}+6} .
$$

The reactance function corresponding to $Z_{L C}^{\square}(s)$ is $X_{L C}^{\square}(\omega):=Z_{L C}^{\square}(j \omega) / j$ and it is depicted in Figure 2. The first canonic Foster's realization of $Z_{L C}^{\square}(s)$ with minimum number of nodes, noncommon-ground capacitors and inductors is depicted in Figure 3. Thereon are denoted the normalized values of $L C$ parameters. 


\section{Conclusions}

In Figure 4, it is depicted the first canonic Foster's realization of driving-point impedance $Z_{L C}^{\square}(s)$ from Figure 3 with selected normalization frequency $\Omega=10^{6}[\mathrm{rad} / \mathrm{s}]$ and selected normalization resistance $R_{0}=10^{3}[\mathrm{k} \Omega]$. The network is excited by a sinusoidal current generator having constant current amplitude and discretely varying frequency $f=\omega /(2 \pi)$ within the range $f \in[0.1,500] \mathrm{kHz}$. If the complex representative of generator current is $\underline{I}_{g}$ and the complex representative of the voltage across its terminals is $\underline{U}_{g}$, then the complex driving-point impedance of the overall $L C$ network is $\underline{Z}_{L C}^{\square}=\underline{U}_{g} / \underline{I}_{g}$. The modulus of $\underline{Z}_{L C^{\prime}}^{\square}$ that is, $\left|\underline{Z}_{L C}^{\square}\right|$ (usually called $L C$ impedance) obtained through PSPICE simulation within the range $f \in[0.1,500] \mathrm{kHz}$ is depicted in Figure 5 .

Now, we will realize $Z_{L C}^{\square}(s)$ by using the proposed Algorithm 2. After LC: $R C$ transformation, we firstly produce the function $\bar{Z}_{R C}(s)=Z_{L C}^{\square}(\sqrt{s}) / \sqrt{s}=1+5 / 2 s+Z_{R C}(s)$, where $Z_{R C}(s)$ is driving-point impedance of $R C$ network which should be expanded into partial fractions as follows:

$$
Z_{R C}(s)=\frac{A_{1}}{s+s_{1}}+\frac{A_{2}}{s+s_{2}}+\frac{A_{3}}{s+s_{3}} \mid \begin{array}{r}
A_{1}=\frac{9}{8}, \quad A_{2}=\frac{3}{4}, \quad A_{3}=\frac{5}{8}, \\
s_{1}=2, \quad s_{2}=4, \quad s_{3}=6, \\
s_{3}>s_{2}>s_{1}>0, \quad \mathbf{S}:=\operatorname{diag}\left(s_{1}, s_{2}, s_{3}\right) .
\end{array}
$$

In step $\left(1^{0}\right)$ of Algorithm 2 we determine the orthogonal matrix $\mathbf{U}$ by using $A_{1}, A_{2}$, and $A_{3}$ (see (2.26) and (5.4))

$$
\mathbf{U}=\left[\begin{array}{ccc}
\sqrt{\frac{2}{5}} & -\sqrt{\frac{3}{5}} & 0 \\
\sqrt{\frac{3}{20}} & \sqrt{\frac{1}{10}} & -\frac{\sqrt{3}}{2} \\
\sqrt{\frac{9}{20}} & \sqrt{\frac{3}{10}} & \frac{1}{2}
\end{array}\right] .
$$

By assuming $\varepsilon_{1}=8 \sqrt{5} / 3$ in step $\left(2^{0}\right)$, we further easily obtain $C=1, G_{1}=2, G_{2}=$ $4, G_{3}=6$ and $\operatorname{col}(\delta)=\left[\begin{array}{lll}\delta_{1} & \delta_{2} & \delta_{3}\end{array}\right]^{\mathrm{T}}=\left[\begin{array}{lll}2.087 & 0.365 & 0.632\end{array}\right]^{\mathrm{T}}$. In step $\left(3^{0}\right)$ we firstly calculate $\boldsymbol{\delta}\left(\mathbf{U G U}^{\mathrm{T}}\right) \boldsymbol{\delta}$ and then hd margins of its rows (columns),

$$
\boldsymbol{\delta}\left(\mathbf{U G U}^{\mathrm{T}}\right) \boldsymbol{\delta}=\left[\begin{array}{ccc}
13.937 & -0.373 & -1.119 \\
-0.373 & 0.693 & -0.320 \\
-1.119 & -0.320 & 1.439
\end{array}\right], \quad p_{1}=12.444, \quad p_{2}=p_{3}=0
$$

In step $\left(4^{0}\right)$ we calculate the normalized capacitances of common-ground capacitors: $C_{1}=$ 4.355, $C_{2}=0.133$ and $C_{3}=0.400$. Realization of driving-point impedance $Z_{R C}(s)$ by transformerless, common-ground $R C$ network with minimum number of nodes $(=n+1)$, reduced number of inductors and minimum number of common-ground capacitors $(=n)$, begins by realization of conductance matrix $\delta\left(\mathbf{U G U}^{\mathrm{T}}\right) \boldsymbol{\delta}$ which can be accomplished almost by inspection of that matrix [22]. Then, to the $i$ th port of the realized conductance network, it should be connected to the capacitor $C_{i}=\delta_{i}^{2}(i=1,2,3)$. The third port of the overall network 
realizes the $R C$ driving-point impedance $Z_{R C}(s)$, provided that all other ports are left opencircuited. By embedding in the third port a series connection of resistor and capacitor with the normalized parameter values 1 and 2/5, respectively, and by applying $R C: L C$ transformation thereafter, we finally produce noncanonic network which realizes $Z_{L C}^{\square}(s)$ with minimum number of nodes and capacitors and with reduced number of inductors. That network is depicted in Figure 6 whereon are denoted the normalized (dimensionless) values of $L C$ parameters.

In Figure 7, is depicted the noncanonic realization of driving-point impedance $Z_{L C}^{\square}(\mathrm{s})$ from Figure 6 with selected normalization frequency $\Omega=10^{6}[\mathrm{rad} / \mathrm{s}]$ and selected normalization resistance $R_{0}=10^{3}[\mathrm{k} \Omega]$. The network is excited by a sinusoidal current generator with constant current amplitude and with discretely variable frequency $f=$ $\omega /(2 \pi)$ within the range $f \in[0.1,500] \mathrm{kHz}$. If the complex representative of generator current is $\underline{I}_{g}$ and the complex representative of the voltage across its terminals is $\underline{U}_{g}$, then the complex driving-point impedance of the overall LC network is $\underline{Z}_{L C}^{\square}=\underline{U}_{g} / \underline{I}_{g}$. The modulus of $\underline{Z}_{L C}^{\square}$, that is, $\left|\underline{Z}_{L C}^{\square}\right|$ (usually called LC impedance) obtained through PSPICE simulation within the range $f \in[0.1,500] \mathrm{kHz}$ is depicted in Figure 8. Since $L C$ networks in Figures 4 and 7 are intentionally designed to be equivalent, then their driving-point impedances $\left|\underline{Z}_{L C}^{\square}\right|$ must have the same variations in frequency, as can be verified from Figures 5 and 8 qualitatively and more precisely by using numerical results of simulation.

A novel procedure for explicit construction of entries of real symmetric matrices with assigned spectrum is developed by using a group of four types of canonic, secondorder, orthogonal transformations. It has been also shown that the orthogonal modal matrices corresponding to the produced real symmetrix matrices, are either lower or upper Hessenberg with explicitly constructed entries too. Thereafter, the inverse eigenvalue problems of real symmetric matrices with twelve specific types of sign patterns (including hyperdominant one) are explicitly solved providing that the signs of eigenvalues are the same (zeros are permitted) and that they are enumerated such as to establish the increasing or decreasing sequence. It is proved to arise thereof a possibility of explicit solving the inverse eigenvalue problem of symmetric hyperdominant matrices having either uncommitted or assigned nonnegative spectrum. The results obtained are then applied in synthesis of drivingpoint immittance functions of transformerless, common-ground, two-element-kind RLC networks and in generation of their equivalent realizations with minimum number of nodes. The synthesis procedures proposed herein turn the synthesis problem of any immittance function of the two-element-kind RLC network to the synthesis problem of impedance function of a prototype $R C$ network.

\section{References}

[1] L. Mirsky, "Matrices with prescribed characteristic roots and diagonal elements," Journal of the London Mathematical Society, vol. 33, pp. 14-21, 1958.

[2] A. J. Schneider, "Construction of matrices having certain sign-pattern and prescribed eigenvalues by orthogonal transformations," IEEE Transactions on Circuits Theory, vol. 12, no. 3, pp. 419-421, 1965.

[3] H. Hochstadt, "On some inverse problems in matrix theory," Archiv der Mathematik, vol. 18, pp. 201207, 1967.

[4] K. P. Hadeler, "Ein inverses Eigenwertproblem," Linear Algebra and Its Applications, vol. 1, no. 1, pp. 83-101, 1968.

[5] K. P. Hadeler, "Multiplikative inverse Eigenwertprobleme," Linear Algebra and Its Applications, vol. 2, no. 1 , pp. $65-86,1969$.

[6] G. N. de Oliveira, "Matrices with prescribed characteristic polynomial and a prescribed submatrix. III," Monatshefte für Mathematik, vol. 75, no. 5, pp. 441-446, 1971. 
[7] J. A. Dias da Silva, "Matrices with prescribed entries and characteristic polynomial," Proceedings of the American Mathematical Society, vol. 45, no. 1, pp. 31-37, 1974.

[8] G. N. de Oliveira, "Matrices with prescribed characteristic polynomial and several prescribed submatrices," Linear and Multilinear Algebra, vol. 2, no. 4, pp. 357-364, 1975.

[9] L. J. Gray and D. G. Wilson, "Construction of a Jacobi matrix from spectral data," Linear Algebra and Its Applications, vol. 14, no. 2, pp. 131-134, 1976.

[10] S. Friedland, "Inverse eigenvalue problems," Linear Algebra and Its Applications, vol. 17, no. 1, pp. 15-51, 1977.

[11] G. N. de Oliveira, "Matrices with prescribed characteristic polynomial and principal blocks. II," Linear Algebra and Its Applications, vol. 47, pp. 35-40, 1982.

[12] S. E. Sussman-Fort, "The reconstruction of bordered-diagonal and Jacobi matrices from spectral data," Journal of the Franklin Institute, vol. 314, no. 5, pp. 271-282, 1982.

[13] I. Zaballa, "Matrices with prescribed rows and invariant factors," Linear Algebra and Its Applications, vol. 87 , pp. 113-146, 1987.

[14] F. C. Silva, "Matrices with prescribed eigenvalues and principal submatrices," Linear Algebra and Its Applications, vol. 92, pp. 241-250, 1987.

[15] W. B. Gragg and W. J. Harrod, "The numerically stable reconstruction of Jacobi matrices from spectral data," Numerische Mathematik, vol. 44, no. 3, pp. 317-335, 1984.

[16] D. B. Kandić, B. Parlett, B. D. Reljin, and P. M. Vasić, "Explicit construction of hyperdominant symmetric matrices with assigned spectrum," Linear Algebra and Its Applications, vol. 258, pp. 41-51, 1997.

[17] A. J. Schneider, "RC driving-point impedance realization by linear transformations," IEEE Transactions on Circuits Theory, vol. 13, no. 3, pp. 265-271, 1966.

[18] S. E. Sussman-Fort, "Inductor-capacitor one-ports and inverse eigenvalue problems," IEEE Transactions on Circuits and Systems, vol. 28, no. 8, pp. 850-853, 1981.

[19] D. B. Kandić and B. D. Reljin, "Class of non-canonic, driving-point immittance realizations of passive, common-ground, transformerless, two-element-kind RLC networks," International Journal of Circuit Theory and Applications, vol. 22, no. 3, pp. 163-174, 1994.

[20] L. Weinberg, Network Analysis and Synthesis, McGraw-Hill, New York, NY, USA, 1961.

[21] B. D. O. Anderson and S. Vongpanitlerd, Network Analysis and Synthesis: A Modern System Theory Approach, Prentice-Hall, Englewood Cliffs, NJ, USA, 1973.

[22] D. B. Kandić and B. D. Reljin, "The application of polynomial matrix factorization in active network synthesis," The International Journal for Computation and Mathematics in Electrical and Electronic Engineering, vol. 24, no. 4, pp. 1120-1141, 2005. 


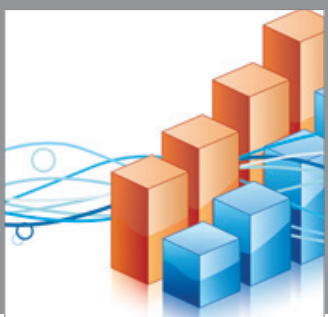

Advances in

Operations Research

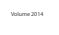

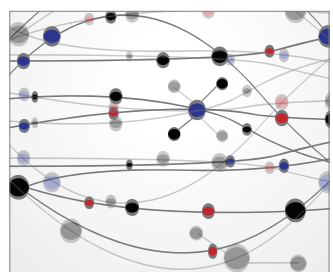

\section{The Scientific} World Journal
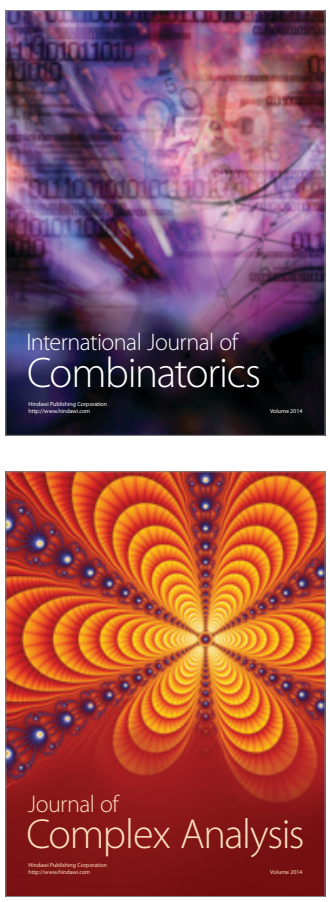

International Journal of

Mathematics and

Mathematical

Sciences
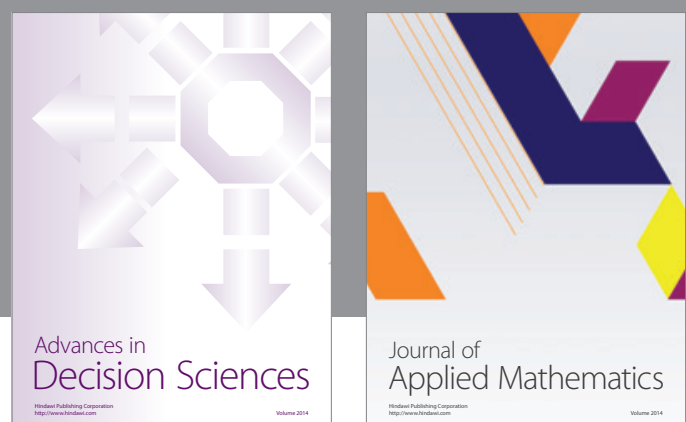

Journal of

Applied Mathematics
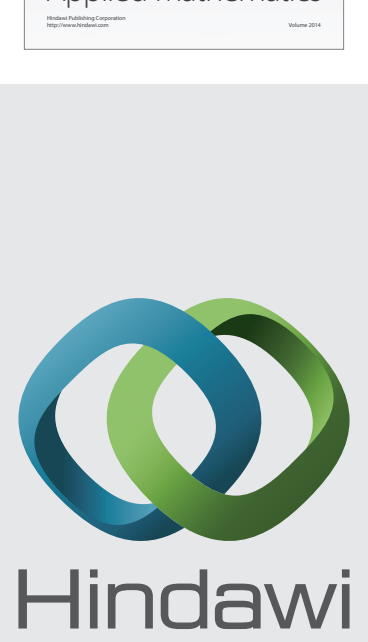

Submit your manuscripts at http://www.hindawi.com
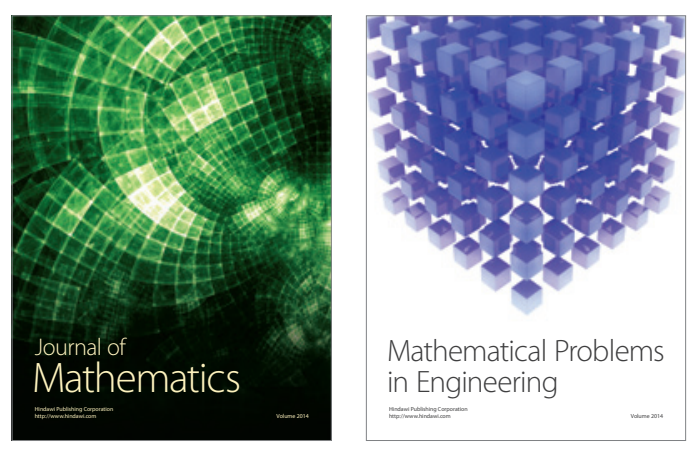

Mathematical Problems in Engineering
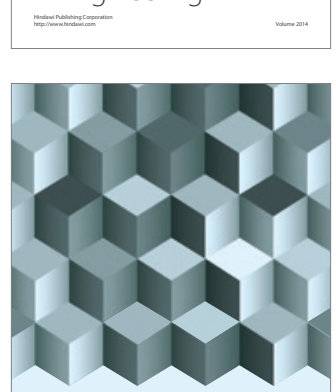

Journal of

Function Spaces
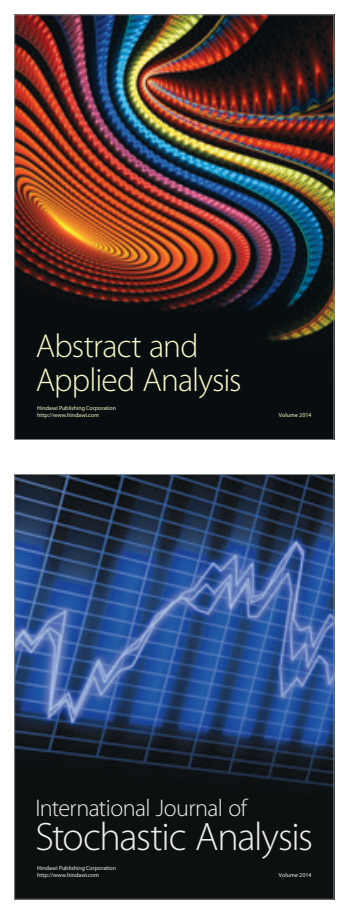

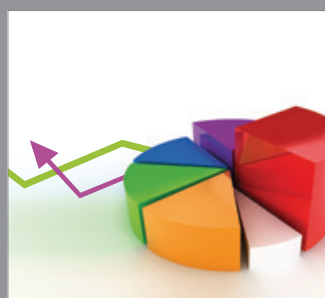

ournal of

Probability and Statistics

Promensencen
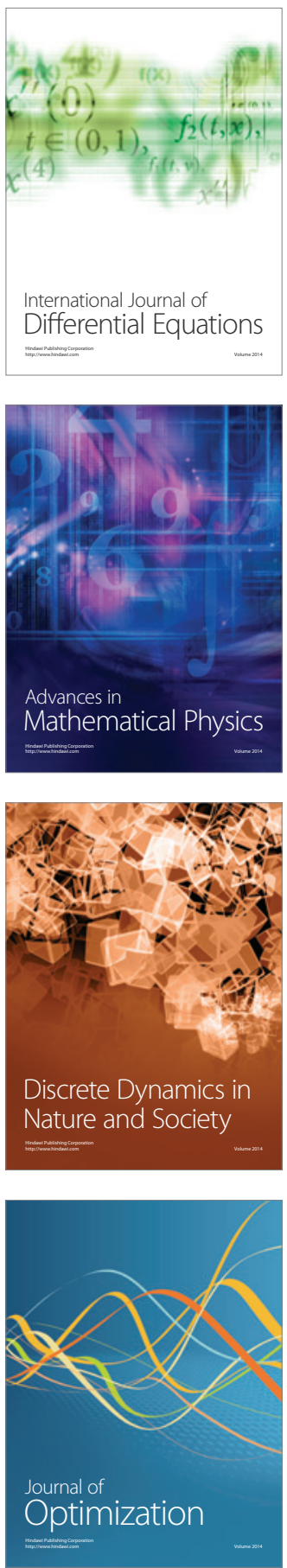